\title{
Genome-wide expression analysis of soybean NF-Y genes reveals potential function in development and drought response
}

\author{
Truyen N. Quach · Hanh T. M. Nguyen • \\ Babu Valliyodan · Trupti Joshi · Dong Xu • \\ Henry T. Nguyen
}

Received: 5 May 2014 / Accepted: 10 December 2014 / Published online: 27 December 2014

(C) The Author(s) 2014. This article is published with open access at Springerlink.com

\begin{abstract}
Nuclear factor-Y (NF-Y), a heterotrimeric transcription factor, is composed of NF-YA, NF-YB and NF-YC proteins. In plants, there are usually more than 10 genes for each family and their members have been identified to be key regulators in many developmental and physiological processes controlling gametogenesis, embryogenesis, nodule development, seed development, abscisic acid (ABA) signaling, flowering time, primary root elongation, blue light responses, endoplasmic reticulum (ER) stress response and drought tolerance. Taking the advantages of the recent soybean genome draft and information on functional characterizations of nuclear factor Y (NF-Y) transcription factor family in plants, we identified $21 \mathrm{GmNF}$ $Y A, 32 \mathrm{GmNF}-Y B$, and $15 \mathrm{GmNF}-Y C$ genes in the soybean (Glycine max) genome. Phylogenetic analyses show that soybean's proteins share strong homology to Arabidopsis and many of them are closely related to functionally
\end{abstract}

Communicated by R. K. Varshney.

T. N. Quach and H. T. M. Nguyen contribute equally to the research.

Electronic supplementary material The online version of this article (doi:10.1007/s00438-014-0978-2) contains supplementary material, which is available to authorized users.

T. N. Quach · H. T. M. Nguyen · B. Valliyodan ·

H. T. Nguyen $(\bowtie)$

Division of Plant Sciences and National Center for Soybean

Biotechnology, University of Missouri, Columbia, MO 65211,

USA

e-mail: nguyenhenry@missouri.edu

Present Address:

T. N. Quach

Field Crop Research Institute, Vietnam Academy of Agricultural

Sciences, Hanoi, Vietnam characterized NF-Y in plants. Expression analysis in various tissues of flower, leaf, root, seeds of different developmental stages, root hairs under rhizobium inoculation, and drought-treated roots and leaves revealed that certain groups of soybean NF-Y are likely involved in specific developmental and stress responses. This study provides extensive evaluation of the soybean NF-Y family and is particularly useful for further functional characterization of GmNF-Y proteins in seed development, nodulation and drought adaptation of soybean.

Keywords NF-Y · Nodulation · Transcription factor · Soybean $\cdot$ Water stress

$\begin{array}{ll}\text { Abbreviations } \\ \text { ABA } & \text { Abscisic acid } \\ \text { DAF } & \text { Days after flowering } \\ \text { ER } & \text { Endoplasmic reticulum } \\ \text { ERN } & \text { Early nodulins } \\ \text { FC } & \text { Fold change } \\ \text { HFM } & \text { Histone fold motif } \\ \text { HMM } & \text { Hidden Markov Model } \\ \text { NF-Y } & \text { Nuclear factor Y } \\ \text { NIN } & \text { Nodule inception }\end{array}$

Present Address:

H. T. M. Nguyen

The Center for Plant Science Innovation, University of Nebraska, Lincoln, NE, USA

T. Joshi · D. Xu

Department of Computer Science, Christopher S. Bond Life Sciences Center, National Center for Soybean Biotechnology and Informatics Institute, University of Missouri, Columbia, MO, USA 
qRT-PCR Quantitative real-time PCR

RPKM Reads per kilobase per million

TF Transcription factor

\section{Introduction}

Nuclear factor Y (NF-Y) is a transcription factor (TF) complex that binds the CCAAT element to regulate gene expression. There are three distinct subunits: NF-YA, NF-YB and NF-YC, which contains evolutionary conserved domains for DNA binding and subunit interaction to form the heterotrimeric NF-Y complex. Subunit assembly is well studied in animals and is conserved in plants (Romier et al. 2003; Calvenzani et al. 2012; Hackenberg et al. 2012). Initially, NF-YB and NF-YC form a dimer complex in cytoplasm and then recruit the third subunit, NF-YA, to form a mature heterotrimemeric NF-Y complex TF (Frontini et al. 2004; Kahle et al. 2005). The binding of NF-Y can either activate or repress transcription of genes (Ceribelli et al. 2008). Sequence analysis of mammalian genome showed $25-30 \%$ of the genes having NF-Y binding sites in their promoters (Mantovani 1998). The CCAAT box is typically found in both forward and reverse orientations between 60 and $100 \mathrm{bp}$ upstream of transcription start site in mammalian genes and may be present in multiple copies with variable distances (Mantovani 1998). In a survey of a promoter sequence population, the CCAAT box element appeared highly associated with TATA-less promoters (Mantovani 1998).

In contrast to animals and yeast where there is a single gene encoding each subunit (Mantovani 1999), there are about 10 genes encoding each subunit in plants (Stephenson et al. 2007; Thirumurugan et al. 2008; Siefers et al. 2009; Liang et al. 2013) with temporal, spatial, universal and organ-specific expression patterns associating with various developmental and environmental response regulations. The roles of certain individuals and NF-Y complexes have been reported in regulation of embryogenesis, cellular signaling, flowering time control, nodulation and nitrogen nutrition and stress tolerance. Arabidopsis NF-YA genes NF-YA1, 3, 5, 6, 8, and 9 have been known to regulate gametogenesis, embryogenesis, seed morphology, and seed germination (Fornari et al. 2013; Mu et al. 2013). Specific members of NF-YB, particularly the LEC 1 group, have been reported to regulate embryogenesis and seed development (West et al. 1994; Parcy et al. 1997; Hawkins and Nakamura 1999; Kwong et al. 2003). LEC1 functions as an integrator of various regulatory events, involving light and hormone signaling, specifically during somatic and early zygotic embryogenesis (Petroni et al. 2012; Laloum et al. 2013) and fatty acid biosynthesis (Mu et al. 2008; Shen et al. 2010; Tan et al. 2011; Mendes et al. 2013). The third subunit, NF-YC, is also important in many developmental regulations. Arabidopsis NF-YC3, NF-YC4 and NF-YC9 physically interact in vivo with both NF-YB2 and NF-YB3 and are required to regulate flowering time.

NF-Y proteins have been reported as a key factor regulating nodulation in the nitrogen-fixing plants and nitrogen assimilation in various plant species. The involvement in nodulation and nitrogen assimilation was identified by the early works on model legume Medicago truncatula for MtNF-YA1 (MtHAP2-1) which controls nodule meristem function (Combier et al. 2006). MtNF-YA1 controls infection thread progression from initial root infection through colonization of nodule tissues (Laporte et al. 2013). Later works on Lotus japonicus identified two NF-Y subunit genes, LjNF-YA1 and LjNF-YB1, which are targeted by nodule inception (NIN) and acted downstream of NIN in the nodule development regulation (Soyano et al. 2013). These two genes are expressed in the root nodule primordia and their protein product can form a NF-Y complex in plant cells. Knockdown of LjNF-YAI inhibited root nodule organogenesis and ectopic expression of the LjNF-YAl and $L j N F-Y B 1$ genes caused abnormal cell division during lateral root development, indicating that the Lotus NF-Y subunits can function to induce cortical cell division to initiate root nodule organogenesis. Recently, the $\mathrm{C}$ subunit from common bean (Phaseolus vulgaris) NF-YC1 was reported to play a key role in the improved nodulation seen by more efficient strains of rhizobia. RNAi-induced reduction of NF-YC1 in hairy roots resulted in the arrest of nodule development and defects in the infection process and the mechanisms appears to involve suppression of cortical cell divisions (Zanetti et al. 2010). Whether PvNF-YC1 functions independently or in combination with other subunits of NF-Y complex, however, has not been known.

In addition to the roles in plant growth and development, NF-Y proteins are important regulators of stress tolerance, especially in response to drought stress (Petroni et al. 2012; Laloum et al. 2013). Overexpression of AtNF-YA5, a strongly drought-induced gene with strong expression in the guard cells, improves drought tolerance and reduces water loss in the leaves ( $\mathrm{Li}$ et al. 2008). In contrast to the overexpression of AtNF-YA5, the nfya5 knockout plants and plants overexpressing miR169a showed enhanced leaf water loss and were more sensitive to drought stress than wild-type plants. In soybean, GmNF-YA3 is induced by various stress treatments. The Arabidopsis transgenic plants overexpressing GmNF-YA3 show reduced leaf water loss and enhanced drought tolerance through regulating the common drought-responsive genes ( $\mathrm{Ni}$ et al. 2013). In rice, the transgenic plants overexpressing HAP2E (NFYA), a gene responsive to wounding, are tolerant to the pathogen Xanthomonas oryzae pv. oryzae, drought and salinity stresses, and improve photosynthesis and tiller 
numbers (Alam et al. 2014). Drought tolerance regulation is also reported in NF-YB subgroup. Overexpression of the drought-inducible NF-YB results in improved performance under drought conditions in Arabidopsis, maize and poplar (Nelson et al. 2007; Han et al. 2013). The transgenic plants have significant improvement in number of stress-related parameters, including chlorophyll content, stomatal conductance, leaf temperature, reduced wilting, maintenance of photosynthesis and the seed yield under water-limited conditions (Nelson et al. 2007). Further characterizations of drought-inducible NF-Y genes will facilitate genetic engineering for improvement of drought tolerance in crop plants.

Soybean is one of the most important crops for its nutrient sources for human and animal consumption, and potential biofuel and economic values. Extensive research has focused on oil seed development (Severin et al. 2010) and plant and symbiotic microbe interaction (Libault et al. 2010), making soybean an ideal plant species for both basic and applied research. Taking the advantage of broad understanding of the recent functional genomics of plant NF-Y genes and the current draft of soybean genome (Schmutz et al. 2010), we attempted to characterize the soybean NF-Y gene family. Together with analysis of NF-Y family in growth and development, we included an analysis of the family members in response to drought and Bradyrhizobium japonicum treatments which are particularly important to identify candidate genes involved in drought tolerance and nodulation and nitrogen fixation in soybean. Our study of transcript expression of soybean NF-Y gene families, therefore, would provide an initial step in understanding gene function that may facilitate functional characterization and genetic engineering to increase the efficiency of soybean in response to drought and $B$. japonicum. In this study, we identified and investigated 68 NF-Y (21 GmNFYA, 32 GmNF-YB, 15 GmNF-YC) and 13 NC2 genes for their expression patterns in soybean tissues and in response to drought stress. Expression analysis showed that there are strong associations between gene expression patterns and homology-based predicted functions, allowing us to assign putative functions to the individual gene groups in regulation of plant growth, development and adaptation to drought stress.

\section{Materials and methods}

In silico identification of soybean NF-Y family members

All soybean protein sequences were downloaded from the Phytozome biomart, gene model V9.1 (http://www.phytozome.net/search.php) using PFAM ID PF02045 (CBFB/
NFYA) and PF00808 (CBFD_NFYB_HMF) and KOG ID KOG0869, KOG0871, KOG1561, KOG1657 and KOG1659, for CCAAT-binding factor subunit A (HAP2), CCAAT-binding factor subunit B (HAP3), CCAAT-binding factor subunit $C$ (HAP5), Class 2 transcription repressor $\mathrm{NC} 2 \beta$ subunit (Dr1) and Class 2 transcription repressor NC2 $\alpha$ subunit (DRAP1), as queries. We used the updated classification (Petroni et al. 2012) of plant NF-Y, NC2 (DR1 and DRAP1) and Dpb3/Dpb4 from Arabidopsis, rice and wheat (both full-length and domain sequences) to classify the retrieved soybean proteins. This updated functional classification has corrected some of the previously identified genes in Arabidopsis, wheat and rice (Stephenson et al. 2007; Thirumurugan et al. 2008; Siefers et al. 2009) by re-grouping some of the previously annotated NF-YB and NF-YC genes to NC2 $\beta, N C 2 \alpha$ and Dpb3/Dpb4 (Petroni et al. 2012). Local BLAST was performed with a threshold of E-10 to classify individuals into functional groups.

Retrieved sequences were aligned using CrustalW with a gap open cost of 10.0 and gap extension cost of 1.0. Phylogenetic tree was constructed by MEGA5 software (Tamura et al. 2011) using neighbor-joining method (Poisson model with uniform rates among sites) and the relationship was estimated using a bootstrap analysis with 1,000 replicates. MultiExperiment Viewer TM4 software (Aryee et al. 2009) was used to generate heat map and hierarchical clustering analysis results.

\section{Plant materials and drought treatment}

Soybean cultivar Williams 82 was grown in greenhouse (28/20 ${ }^{\circ} \mathrm{C}$ day/night temperature, 14-h day-length photoperiod, $800 \mu \mathrm{mol} \mathrm{m}{ }^{-2} \mathrm{~s}^{-1}$ light intensity and $60 \%$ humidity) with a density of four plants per 1-gallon pot, containing a mixture of 1 sand: 1 turface. The plants were watered every 2 days. Drought treatments started when the plants reached the first leaf stage (V1-first fully open trifoliolate leaf stage, day 14th) by withholding watering and the plants were harvested when the desired stem water potential was reached. There were three drought treatments with stem water potentials of -0.5 (day 18th), -1.0 (day 22th) and $-1.5 \mathrm{MPa}$ (day 34th) for drought and the corresponding control treatments had a stem water potential of $\sim-0$. $2 \mathrm{MPa}$. To measure stem water potential, the stems were cut at the hypocotyl segment and immediately measured for water potential using a pressure chamber (PMS Instrument Co. Albany, CA, USA) at around 4Am. Predawn tissue collection was selected because it was considered equivalent to whole soil water potentials. Three biological replicate experiments were conducted following a complete randomized design. Each replicate consisted of samples pooled from three plants. 
RNA isolation, cDNA synthesis and mRNA expression analyses for drought responses

Each frozen tissue collected from the three-biological repeat drought experiment was separated into two technical repeats and grinded in liquid nitrogen. RNA was extracted using Trizol (Invitrogen Inc.). RNA integrity was verified by electrophoresis using $1 \%$ agarose in buffer TAE. The RNA was purified from DNA contamination using the Turbo DNA-free DNaseI kit (Ambion, Austin, TX, USA). One microgram of total RNA and the primer mix of oligodT and random hexamer were used to synthesize cDNA using iScript cDNA Synthesis Kit (Bio-Rad, Hercules, CA, USA) in a reaction volume of $20 \mu \mathrm{L}$. Quantitative real-time PCR (qRT-PCR) was performed to quantify mRNA expression under drought conditions. Initially, PRIMEGENS (Srivastava and $\mathrm{Xu}$ 2007) was used to design primers for soybean NF-Y gene family and all the obtained sequences were manually verified for specific alignment by Blast against the soybean genome database housed in Phytozome to have the final primer list (Supplementary Table S2). Primer pairs with acceptable PCR efficiency and the unique melting disassociation curve were used for qRT-PCR. All reference genes (Supplementary Table S3) were selected based on recommendations from current literatures (Jian et al. 2008; Libault et al. 2008; Hu et al. 2009; Le et al. 2012). qRT-PCR results were analyzed using a common PCR efficiency for each primer obtained from linregPCR analysis software (Ramakers et al. 2003). The obtained efficiencies were consistently higher than 1.8 with average of about 1.95 for whole primer sets. GeNorm (Vandesompele et al. 2002) was used to test the stability (M index) to determine a set of reference genes to be used to normalize individual expression data. In our experiment, we calculated relative expression by normalizing data to the four best reference genes: IDE, UNK1, UNK2 and ACT (Supplementary Figure S1). Fold change of gene expression was calculated only when there was a significant difference in means of relative gene expression between the drought and control samples from a Student $T$ test.

Tissue-specific expression and responses to B. japonicum treatments

Data were retrieved from previously reported datasets (Libault et al. 2010; Severin et al. 2010) and detailed experimental procedures were described therein and briefly summarized below. Tissue-specific expression in 14 tissues was performed on soybean genotype P-C609-45-2-2 (Severin et al. 2010). The soybean plants were growing in pots containing B. japonicum-inoculated soil and fullnutrient fertilizer (Osmocote 14-14-14) in growth chambers under photoperiod of $14 / 10 \mathrm{~h}$, thermocycle of $22 / 10^{\circ} \mathrm{C}$, relative humidity of 50-60\%, and light intensity of 550$740 \mu \mathrm{E} \mathrm{m}^{-2} \mathrm{~s}^{-1}$. Samples were pooled from a minimum of three plants. Young leaf tissue samples were collected at flowering stage and pods and seeds were harvested by seed weight and pod lengths. The $1-\mathrm{cm}$ pod was approximately harvested at 7 days after flowering (DAF), and the 4- and 5-cm pods were harvested at 10-13 DAF and 14-17 DAF, respectively, and were divided into seed and pod-shell components. Seed 21-, 25-, 28- and 35-DAF had seed weights between 10 and $25 \mathrm{mg}, 25$ and $50 \mathrm{mg}, 50$ and $100 \mathrm{mg}, 100$ and $200 \mathrm{mg}$, and greater than $200 \mathrm{mg}$, respectively. Root and nodule tissues were from plants grown in growth chambers under 16-h photoperiods and light intensities between 310 and $380 \mu \mathrm{E} \mathrm{m}^{-2} \mathrm{~s}^{-1}$. Root tissue was harvested after 12 days and nodules were harvested at 20-25 days after bacterial inoculation. For analysis of gene expression in root hairs in response to $B$. japonicum treatments, normalized data were retrieved from a previous report (Libault et al. 2010) and the experimental procedures are described briefly below. At least three independent biological replicates were produced to ensure the reproducibility of the plant tissues analyzed. Soybean (cv. Williams 82) seeds were germinated on nitrogen-free $\mathrm{B} \& \mathrm{D}$ agar medium in growth chambers (dark conditions, $80 \%$ humidity, $27^{\circ} \mathrm{C}$ ). Three days after sowing, a $B$. japonicum strain USDA 110 cell suspension or water (mock inoculation) was sprayed on the seedlings and the treated seedlings were returned to the original growth chamber for incubation. Root hair cells were isolated after 12, 24 and $48 \mathrm{~h}$ of bacterial inoculation. For each condition, similar quantities of total RNA isolated from three independent biological replicates were pooled together to synthesize cDNA which were then ligated to Solexa adaptors. Complementary DNAs were sequenced using Solexa platform and data were analyzed using Illumina Genome Analyzer II, and the data were analyzed and normalized to reads per kilobase per million (RPKM).

\section{Results}

Identification of NF-Y genes from soybean

BLAST searches using functionally characterized NF-Y domains as queries resulted in 134 sequences belonging to 81 gene loci. We used the primary transcript as a representative from each gene for further analysis of transcriptome. The final gene list is divided into four groups: $21 \mathrm{GmNF}-$ YA, 32GmNF-YB, 15GmNF-YC, 11 NC2 and 2 Dpb3 proteins (Table 1). BLAST searches using Arabidopsis protein sequences as queries resulted in similar classified groups (Supplementary Table S1). NC2 and Dpb3 proteins share the histone fold motif (HFM), but lack of essential amino acids for subunit interactions (as discussed later); therefore, 
were excluded from further functional analysis relating to NF-Y gene families.

For verification purposes, soybean NF-Y protein sequences were downloaded from three current databases of soybean transcription factors. The first database, SoyDB (http://soykb.org/), provides the predicted transcription factors from InterProScan searches against 11 databases integrated in InterPro to find transcription factor domains (Wang et al. 2010). The second soybean transcription database, SoybeanTFDB (http://soybeantfdb.psc.riken.jp/), used Hidden Markov Model (HMM) of PFAM to search against the modeled proteome data of annotated genes in Glymal from Phytozome database with a threshold of $E<1$ e-5 and a verification from domain searches using InterProScan and Blastp for putative homologous genes (Mochida et al. 2010). The third transcription factor database, PlantTFDB (http://planttfdb.cbi.pku.edu.cn/), used 48 HMM-Pfam profiles and 16 additional HMM constructed from GenPept and Swiss-Prot protein databases to construct $64 \mathrm{TF}$ families in Arabidopsis and other plant species (Guo et al. 2008). Alignment analysis of these obtained sequences resulted in a number of genes incorrectly classified to NF-YB and NF-YC subfamilies (data not shown) mainly due to the fact that the classification did not have a separate description and classification for NF-YB, NF-YC, NC2 or Dpb group, but instead, have a common description for these two groups with a name NF-YB/HMF domain. Given the critical differences among NC2, Dpb and NF-Y groups, it is necessary to annotate them accurately for the updated research.

Tissue differential expression and drought and $B$.

japonicum inducibility of NF-Y genes

Publicly available gene expression in 14 soybean tissue types including leaf, flower, pod, root, nodules and various stages of seed development (Severin et al. 2010) for 54 soybean NF-Y genes was retrieved and used to construct a hierarchical clustering heat map (Fig. 1). The data show that there are some preferences in expression of each gene subfamily in certain plant tissues. A small group of genes highly specific to 1- to 3-week-old seeds (R3 growthstaged seeds) belong strictly to NF-YC (GmNF-YC05, GmNF-YC09, GmNF-YC10 and GmNF-YC11) while majority of seed deferential genes with stronger expression belong to NF-YA and NF-YB genes. A large group of 14 genes strictly to NF-YA and NF-YB are highly specific to root and/or nodules. We also included the data retrieved from a mRNA expression analysis of root hairs in response to treatments of $B$. japonicum (Libault et al. 2010) which clearly show that all the genes that are strongly induced (10- to 63-fold change) by B. japonicum in root hair are nodule-specific GmNF-YA members: NF-YA01, NF-YA03,
NF-YA10 and NF-YA21 (Fig. 1; Supplementary Figure S2). There are several smaller gene groups which are more universal in expression and can be sub-grouped due to slight preference in green organs, flowers and leaves. Members of this group belonging to all families of NF-Y and NC2 including NF-YC14, NF-YB13, NF-YA07 and NF-YA20 are differentially expressed in green organs (leaves and pods) while NF-YB02, NF-Y06, Dpb3-1, Dpb3-2 and NC2 33 differentially expressed in leaves.

To identify candidate genes for drought tolerance in soybean, we performed transcript profiling for the soybean CCAAT box gene family using qRT-PCR for root and leaf samples of three drought treatments of $-0.5,-1.0$ and $-1.5 \mathrm{MPa}$ predawn stem water potentials. We were able to amplify and quantify expression of total 75 genes (Supplementary Table S2). Using a fold-change cut-off of three, 30 genes were found to respond to drought treatment in soybean leaf and root samples (Fig. 2) and NF-YA members appear to be more drought responsive. Most seed differentially expressing genes are drought induced while the root/nodule-specific genes have tendency to be drought repressed (Fig. 1).

Domain structure and putative function of soybean NF-Y subunits

\section{GmNF-YA}

Using the sequences of known NF-YA/HAP2 proteins, we could identify $21 \mathrm{GmNF}-Y A$ genes from the soybean genome. Soybean NF-YA proteins have variable length from 206 to 347 amino acids, a comparable range with M. truncatula (Laloum et al. 2013). There is no similarity between NF-YA and other transcription families except some homology to the CCT domain of the flowering time regulator CONSTANS (Wenkel et al. 2006). NF-YA proteins are characterized by a conserved sequence that includes a protein interaction domain which can bind to the combined surface of NF-YB/NF-YC heterodimers (Hackenberg et al. 2012) and a DNA-binding domain (Fig. 3). The subunit interaction domain consists of 20 amino acids and alpha helix that is important for interaction with NF-YB and NF-YC subunits (Mantovani 1999). The DNA-binding domain of 21 amino acids is separated from the subunit interaction domains by a relatively conserved linker. Most mammalian and yeast functionally required amino acid residues (Maity and de Crombrugghe 1992; Xing et al. 1993) are present in the soybean NF-YA proteins. There are some alterations for alanine $\left(\mathrm{A}_{4}\right.$ and $\left.\mathrm{A}_{16}\right)$ and leucine $\left(\mathrm{L}_{11}\right)$ in soybean which can be replaced by hydrophobic residues (Maity and de Crombrugghe 1992) and arginine $\left(R_{9}\right)$ is commonly replaced by glycine and alanine which is usually seen in plant NF-YA (Siefers et al. 


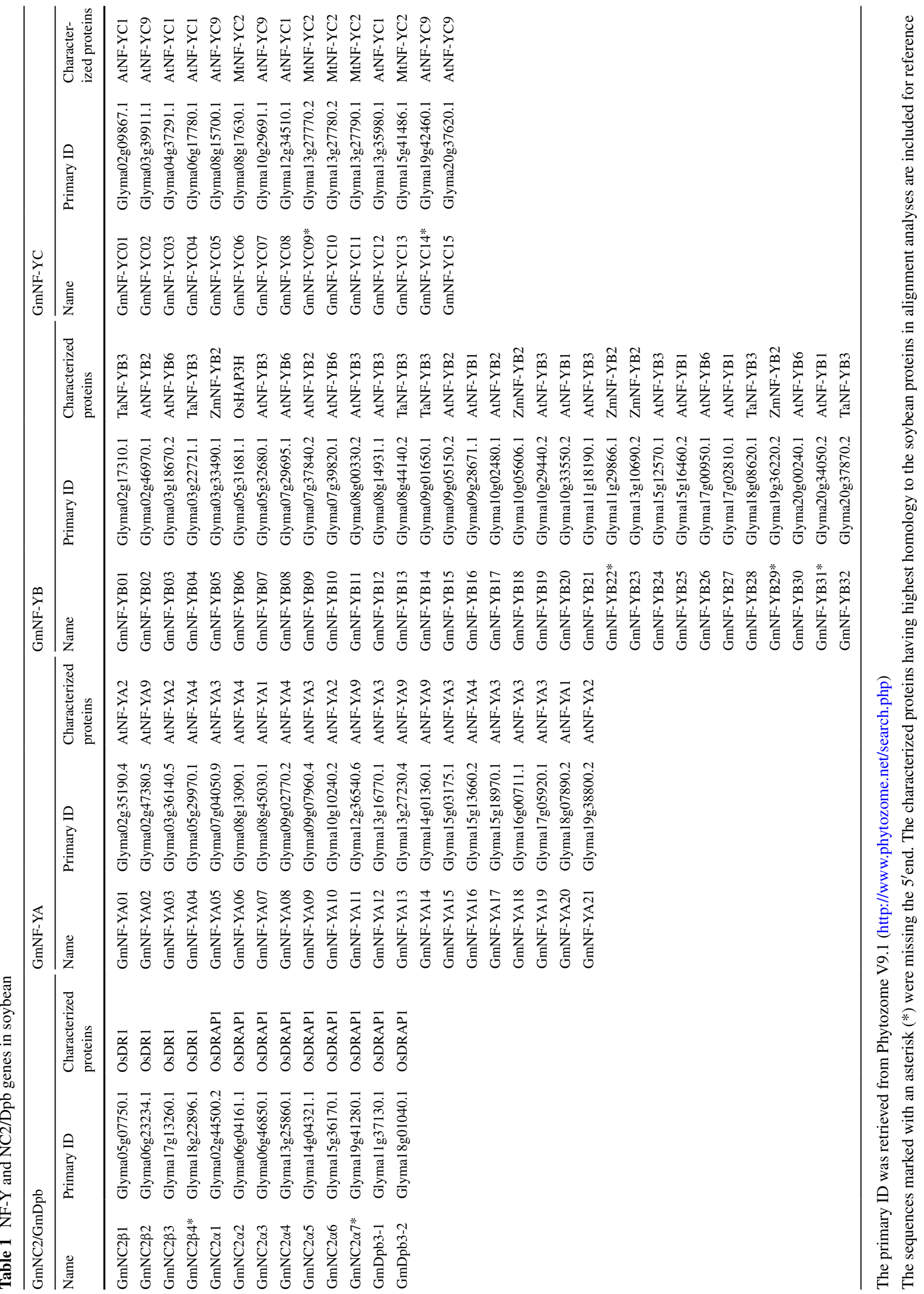


2009). Among soybean NF-Y proteins, only NF-YA subunits possess a nuclear localization signal (Kahle et al. 2005; Thon et al. 2010) with the presence of all three clusters of basic residues (Fig. 3). The N-terminus of NF-YAs is acidic and rich in glutamines and serine/threonine at $\mathrm{N}$-termini, and loosely conserved and might function as an activation domain (Mantovani 1999).

A number of functional groups can be putatively assigned (Fig. 4). Group A consists of GmNF-YA01, GmNF-YA03 and GmNF-YA10 which are expressed highly in nodules and have sequences highly similar to the M. truncatula MtNF-YA1 (Combier et al. 2006), Lotus japonicus LjNF-YA1 (Soyano et al. 2013) and Arabidopsis AtNF-YA2 (Zhao et al. 2011), which are important in nodule development and nitrogen nutrition. These three nodule-specific genes are also induced strongly by inoculation with B. japonicum (Fig. 1). Group B contains the largest number of soybean GmNF-YA which have differential expression in flower and seeds and share sequence similarity with at least five functionally characterized proteins relating to $\mathrm{ABA} /$ dehydration signaling in seed development. Of these, GmNF-YA12 [known as GmNF-YA3 (Ni et al. 2013)] is induced by ABA, $\mathrm{NaCl}$, PEG and cold treatments and controlled by miRNA169 to regulate drought tolerance in the Arabidopsis model (Ni et al. 2013). Genes in group B have diverse functions: AtNF-YA3 and AtNFYA8 which regulate embryogenesis (Fornari et al. 2013) and AtNF-YA5 and AtNF-YA6 which regulate ABA and blue light signaling and drought tolerance (Warpeha et al. 2007; Li et al. 2008), and AtNF-YA4 which controls ER stress tolerance and flowering time (Wenkel et al. 2006; Liu and Howell 2010). In contrast, genes in group C might be particularly functioning in ABA-related seed development and maturation which are relevant to the functions of Arabidopsis AtNF-YA1 and AtNF-YA9 (LevesqueLemay et al. 2003; Wenkel et al. 2006; Li et al. 2013; Mu et al. 2013). Sequence analysis predicted that 12 soybean GmNF-YA genes are targeted by miR169d and miR169e (TAG_5193213 and TAG_1196776, respectively). Expression miR169d was not associated with any of the NF-YA; however, expression of miRNA169e was negatively associated with GmNF-YA02, GmNF-YA12, GmNF-YA14 and GmNF-YA20 (data not shown). GmNF-YA12 has been shown to be cleaved by miR169e in a coexpression study in Nicotiana benthamiana and 5' RACE assays (Ni et al. 2013).

$N F-Y B$

The NF-YB subunits are characterized by the conserved NF-YA and NF-YC interaction domains and a DNAbinding domain (Fig. 5). Sequence structure of NF-YB is related to histone fold motif of H2B histone (Mantovani
1999). Histone has a conserved domain of 65 -amino acid HFM with low sequence identity but high structural resemblance (Arents and Moudrianakis 1995). In Arabidopsis the combined surface of the NF-YB/NF-YC heterodimer is required for the interaction with NF-YA subunit and the translocation of the complex into nucleus (Hackenberg et al. 2012).

The residues required for the subunit interaction and DNA binding (Xing et al. 1993; Sinha et al. 1996) are highly conserved among GmNF-YB proteins except for relatively few alterations by similar functional residues (Fig. 5). Arginine $\left(R_{55}\right)$ and aspartate $\left(D_{65}\right)$ are suggested to have a crucial role in protein interaction due to possession of an important intramolecular hydrogen bond within the HFM (Romier et al. 2003; Hackenberg et al. 2012). These arginine-aspartate pairs are highly conserved through evolution in almost plants and animal species and are important in subunit interactions between NF-YB and NF-YC (Romier et al. 2003; Hackenberg et al. 2012). They are present in all soybean NF-YB proteins except GmNF-YB16 and GmNF-YB22 which have deletions in this domain and might not be able to form the NF-Y complex. Given the number of the GmNF-YB is almost three times while the numbers of GmNF-YA and GmNF-YC are about two times of corresponding genes in Arabidopsis, it is likely that the GmNF-YB proteins have gone through largest functional alteration.

Analysis of domain sequence of Arabidopsis NF-YB proteins reveals that the translocation of plant NF-Y complex into nucleus follows the piggyback transport model (Hackenberg et al. 2012) in which NF-YB and NF-YC heterodimer is formed first by head-to-tail annealing of their HFMs (Thon et al. 2010). A disulfide bond between two cysteines of NF-YB $\left(\mathrm{C}_{33}\right.$ and $\left.\mathrm{C}_{37}\right)$ controls the heterodimerization of two subunits and further controls the spatial distribution of subunits and subcellular localization of the NF-Y complex upon a redox state. In soybean, all NF-YBs apparently lack the critical cysteine at position 33 and cannot form the disulfide bond (Fig. 5) which resembles the model of nuclear translocation of NF-Y complex in Arabidopsis (Hackenberg et al. 2012). Therefore, the redox-regulated assembly of NF-YB and NF-YC of human and Aspergillus nidulans is not present in the soybean NF-Y.

NF-YB proteins can be putatively assigned to several functional groups (Fig. 6). Group D contains proteins that are closely related to the wheat TaNF-YB3 which regulates photosynthesis (Stephenson et al. 2011), Arabidopsis AtNF-YB2 and AtNF-YB3, and the rice OsHAP3S which controls flowering time control (Kumimoto et al. 2008; Wei et al. 2010). Surprisingly, many of the soybean genes are highly expressed in nodule suggesting that they may function in nodulations and nitrogen nutrition. Members of group E including GmNF-YB03, GmNF-YB10, 


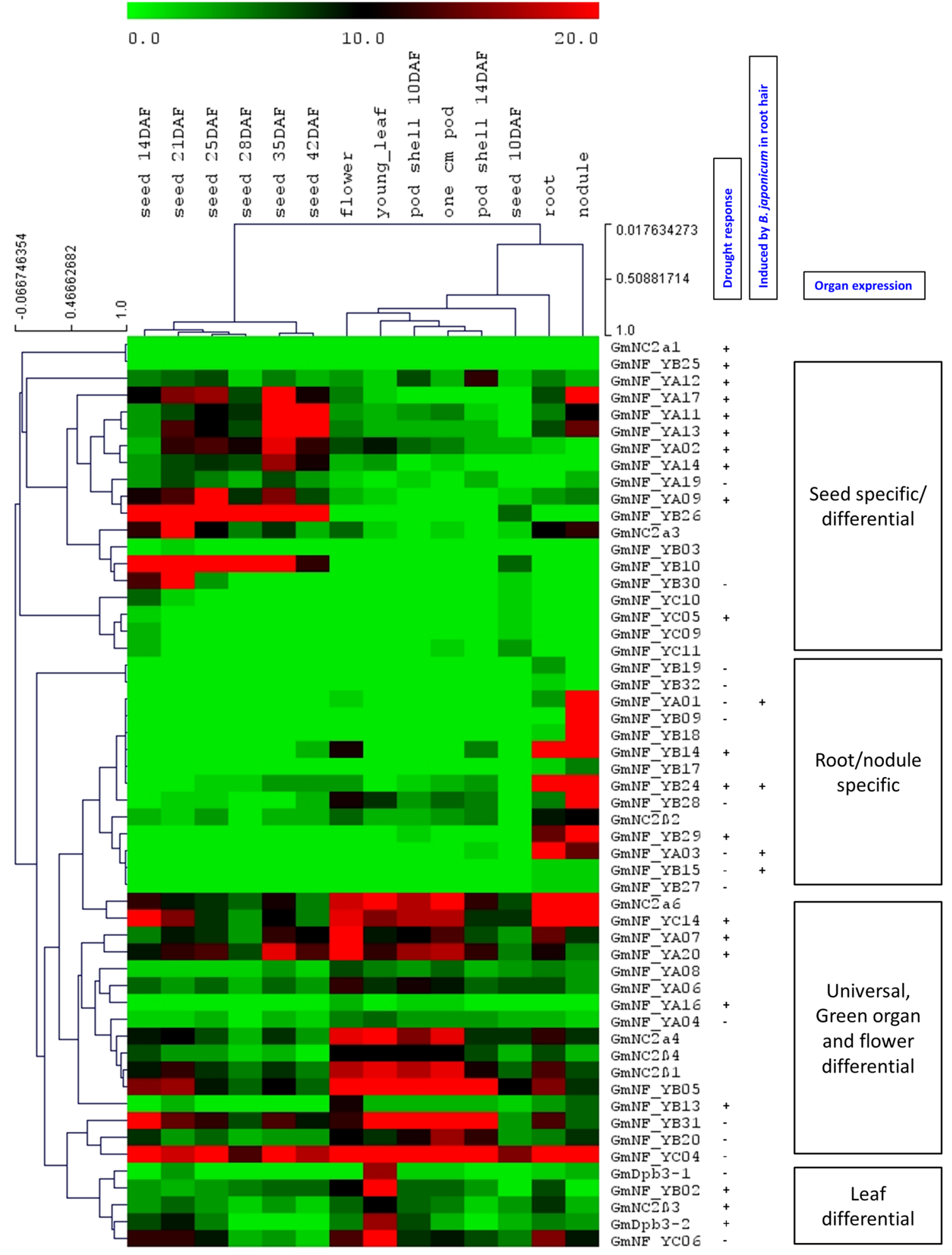

Fig. 1 Expression of soybean NF-Y in various tissues, drought stress and rhizobium inoculation. Data of 54 GmNF-Y genes were retrieved from the normalized RNA sequencing databases (Libault et al. 2010; Severin et al. 2010) for clustering analysis of tissue-specific and stress-response expression. For drought and rhizobium treatments $(+)$ and (-) indicate up and down gene expression in both root and leaf tissues tested, compared to non-stress condition. The color bar represents number of RPKM (color figure online) 


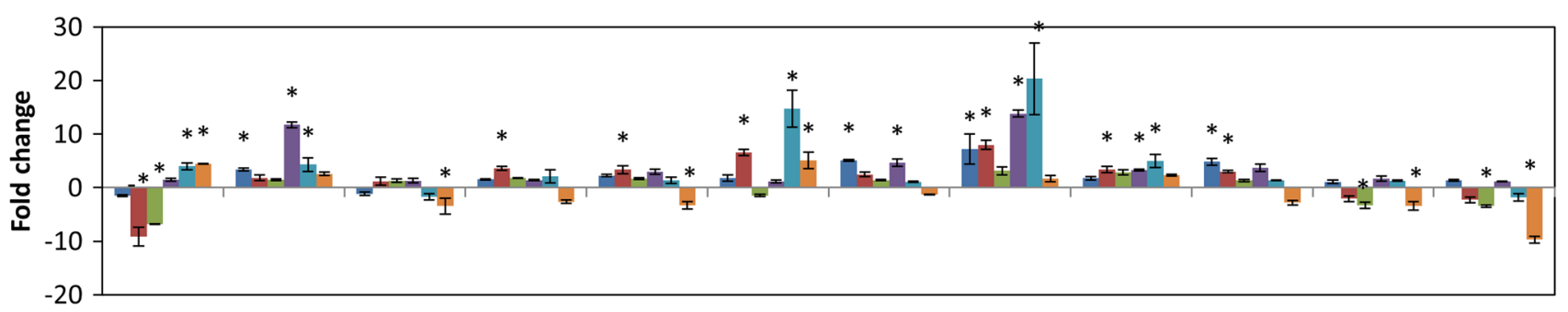

NF-YA01 NF-YA02 NF-YA04 NF-YA09 NF-YA11 NF-YA12 NF-YA13 NF-YA14 NF-YA16 NF-YA17 NF-YA19 NF-YA21
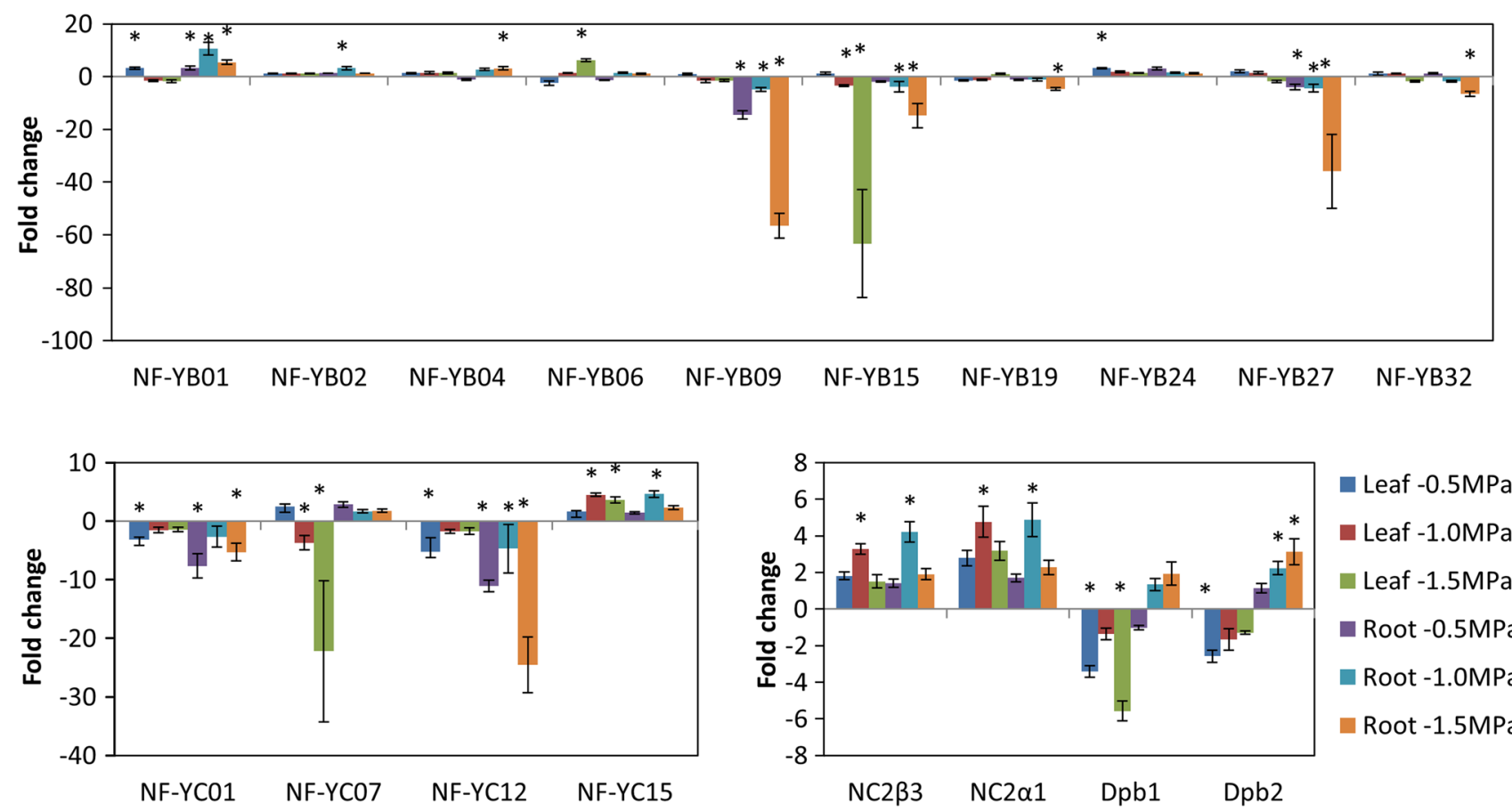

Leaf $-0.5 \mathrm{MPa}$

Leaf -1.0MPa

Leaf -1.5MPa

Root $-0.5 \mathrm{MPa}$

Root -1.0MPa

Root $-1.5 \mathrm{MPa}$

Fig. 2 Expression of highly drought-responsive soybean NF-Y genes. These genes have at least threefold-changed (FC) expression compared to the non-stress controls. Significant FCs were marked with an asterisk from three biological repeats

GmNF-YB26 and GmNF-YB30 share homology to LEC1 and LEC1-like (AtNF-YB9 and AtNF-YB6). All these sequence have an aspartate at position $28\left(\mathrm{D}_{28}\right)$ which is critical for LEC1 function and is not present in all other members of NF-YB proteins (Lee et al. 2003) indicating the unique function of this LEC1 group in embryogenesis and ABA signaling (Lotan et al. 1998; Kwong et al. 2003; Warpeha et al. 2007). LEC1 and LEC1-like orthologs have a conserved function in fatty acid biogenesis and seed oil production in Brassica napus (Tan et al. 2011; Mu et al. 2013) and maize (Shen et al. 2010). Group F composed a cluster of root/nodule differentially expressed NF-YB genes homologous to the L. japonicus protein LjNF-YB1, which together with LjNF-YA1 regulates division of cortical cells, an initial step in root nodule organogenesis (Soyano et al. 2013). Surprisingly, majority of nodule differential and rhizobium inducible genes in this group are drought repressed. Their close Arabidopsis homolog,
$A t N F-Y B 1$, is an important gene regulating drought tolerance (Nelson et al. 2007).

Among three NF-Y subfamilies, only members of plant NF-YB have been identified to involve photosynthesis so far. Rice OsHAP3 genes regulate the expression of nuclear-encoded chloroplast-targeted genes and normal development of chloroplasts (Miyoshi et al. 2003). Downregulation of OsHAP3A, OsHAP3B and OsHAP3C resulted in reduced chlorophyll and degenerated chloroplasts which failed to develop lamella and accumulate starch. These plants showed reduced expression of nuclear-encoded photosynthesis genes. In accordance with this, a wheat TaNF-YB3 was identified to co-express with photosynthetic genes and the transgenic plants showed increased leaf chlorophyll content and photosynthesis (Stephenson et al. 2011). Although close orthologs can be found in soybean, these genes do not belong to a certain phylogenetic tree branch (Fig. 6) making it 


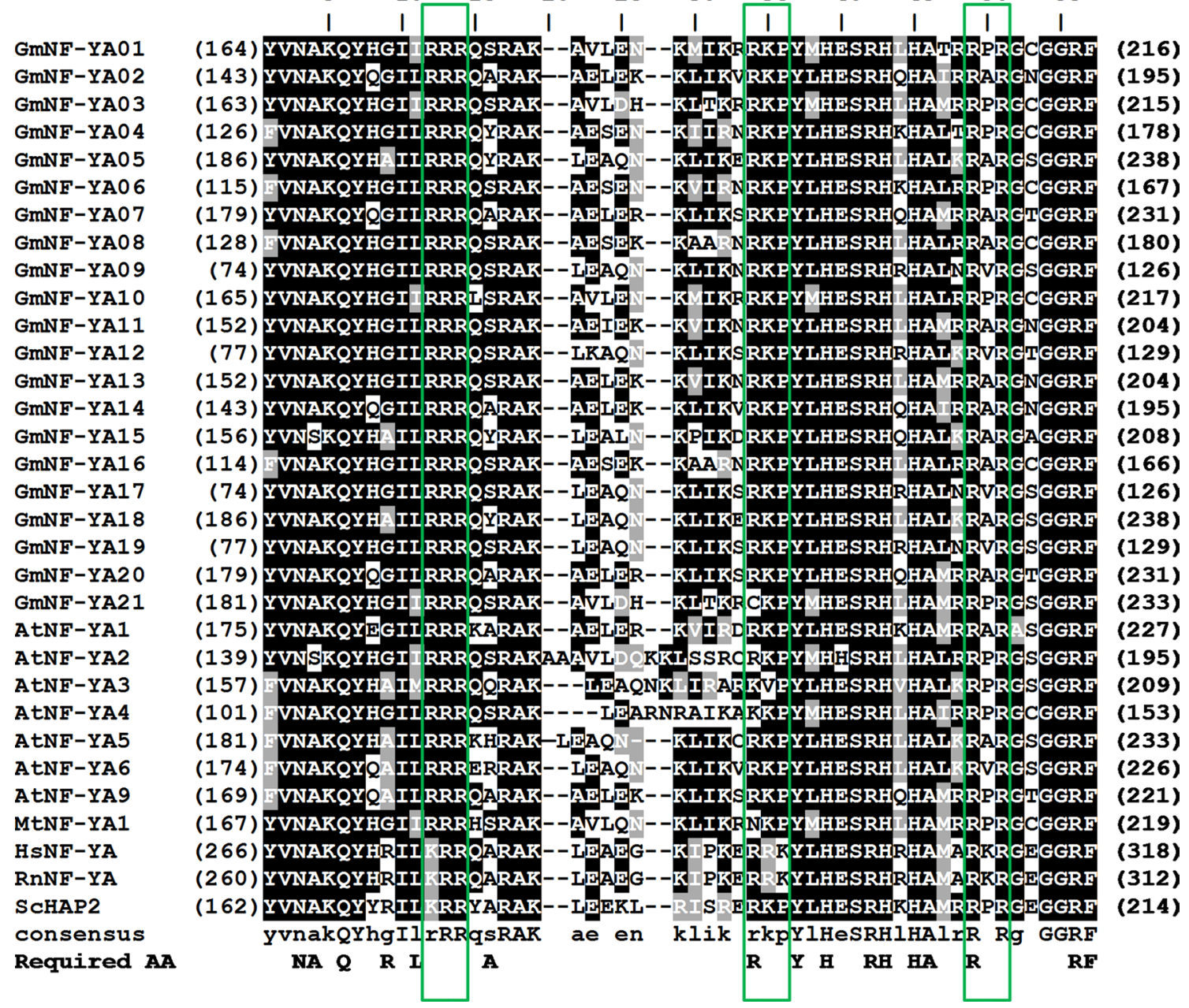

Fig. 3 Alignment of soybean NF-YA domains. The referred sequences are from Arabidopsis (At), Medicago (Mt), human (Hs), mouse (Rn) and yeast $(\mathrm{Sc})$. Numbers in parentheses are the actual amino acid numbers of the start and end of NF-YA domain. Required amino acids

unreliable to predict soybean NF-YB genes functioning in photosynthesis.

\section{$N F-Y C$}

Soybean genome has 15 genes encoding NF-YC subunits which have between 112 and 291 amino acids in length. GmNF-YC possesses of a highly conserved domain for NF-YA and NF-YB interaction (phylogenetic tree bootstrap values are substantially low, Fig. 7) and have structural and amino acid homology to H2A histone (Mantovani 1999). The majority of the required amino acids for the subunit interaction and DNA binding of NF-YC (Kim et al. 1996; Sinha et al. 1996) are highly conserved with minimal replacement of alternative residues of the same
(Required AA) are important for DNA binding and subunit interaction from yeast (Xing et al. 1993) and rat (Maity and de Crombrugghe 1992). The three boxes in green are the basic residual clusters required for nuclear targeting (Peng et al. 1998) (color figure online)

functional properties. The two amino acids arginine $\left(\mathrm{R}_{52}\right)$ and aspartate $\left(\mathrm{D}_{59}\right)$ necessary in stabilizing the structural conformations of NF-YB and NF-YC through the HFMs for mutual interaction between the NF-YB and NF-YC subunits (Hackenberg et al. 2012) are present in most soybean NF-YC, except for GmNF-YC11 (Fig. 7). GmNF-YC genes are expressed in various tissues except a small group of genes which are expressed specific in early seed development stages. Compared to NF-YA and NF-YB subfamily, fewer NF-YC genes showed response to drought treatment with only GmNF-YC15 is induced and GmNF-YC01, GmNF-YC07 and GmNF-YC12 are suppressed (Fig. 2).

The sequences of NF-YC proteins are highly conserved (Fig. 8). The bootstrap supporting for most clades is quite low (generally $<70 \%$ ); making the tree not necessarily 


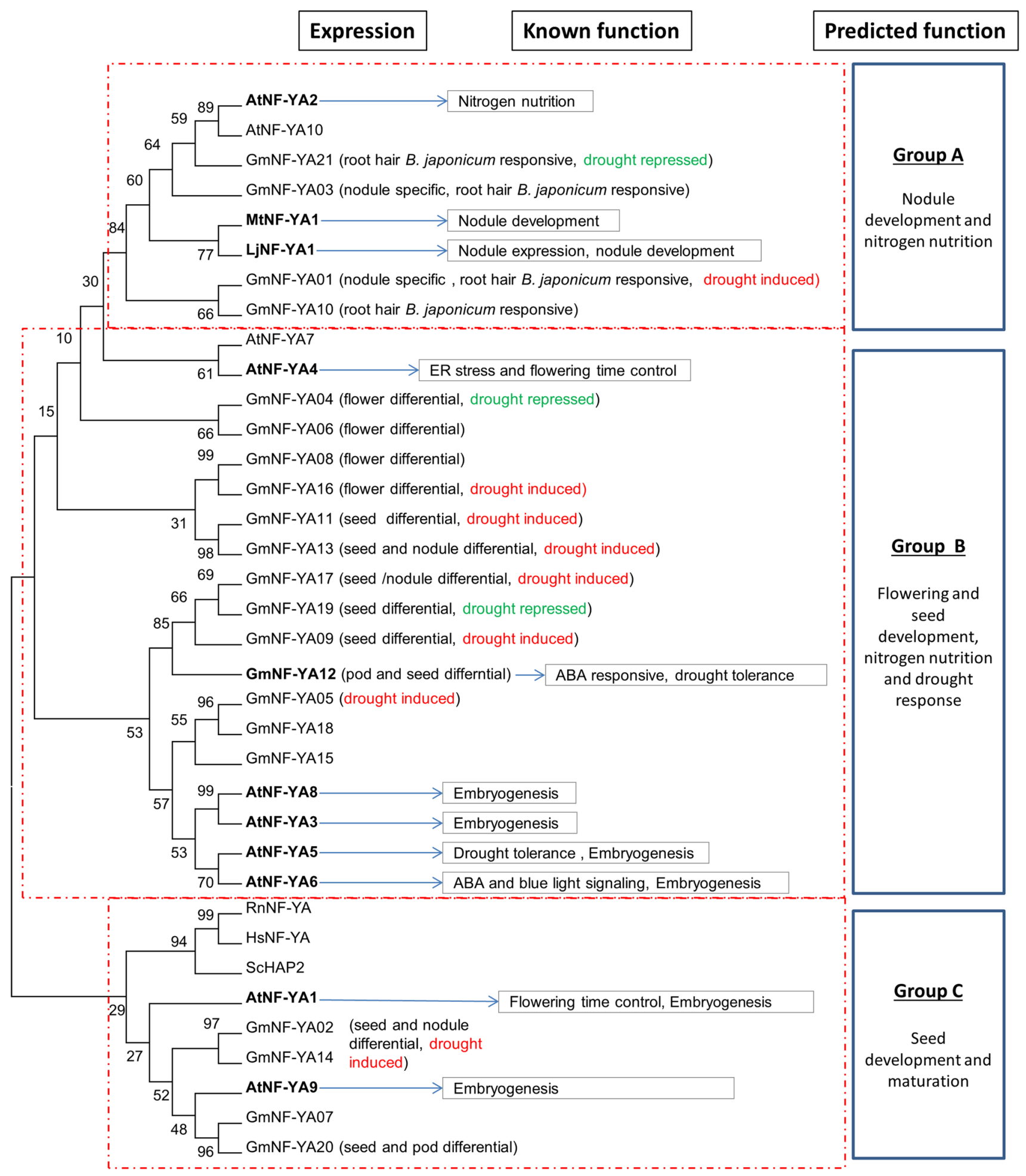

Fig. 4 Phylogenetic tree of soybean NF-YA. The tree was constructed using MEGA5 software (see "Materials and methods") for the NF-YA domain sequences. Bootstrap values (from 1,000 replicates) were shown to represent the reliability of tree branches. The referred sequences are from Arabidopsis (At), Medicago (Mt), L. japonicus $(\mathrm{Lj})$ human $(\mathrm{Hs})$, mouse $(\mathrm{Rn})$ and yeast $(\mathrm{Sc})$. Function prediction for soybean genes were based on gene expression and homology to the referred characterized proteins which are written in bold for AtNF-YA1 (Wenkel et al. 2006; Mu et al. 2013), AtNF-YA2 (Zhao et al. 2011), AtNF-YA3 (Fornari et al. 2013), AtNF-YA4 (Wenkel et al. 2006; Liu and Howell 2010), AtNF-YA5 (Li et al. 2008; Mu et al. 2013), AtNF-YA6 (Fornari et al. 2013; Mu et al. 2013), AtNFYA8 (Fornari et al. 2013), AtNF-YA9 (Mu et al. 2013), MtNF-YA1 (Combier et al. 2006), LjNF-YA4 (Soyano et al. 2013) and GmNFYA12 (Ni et al. 2013) 


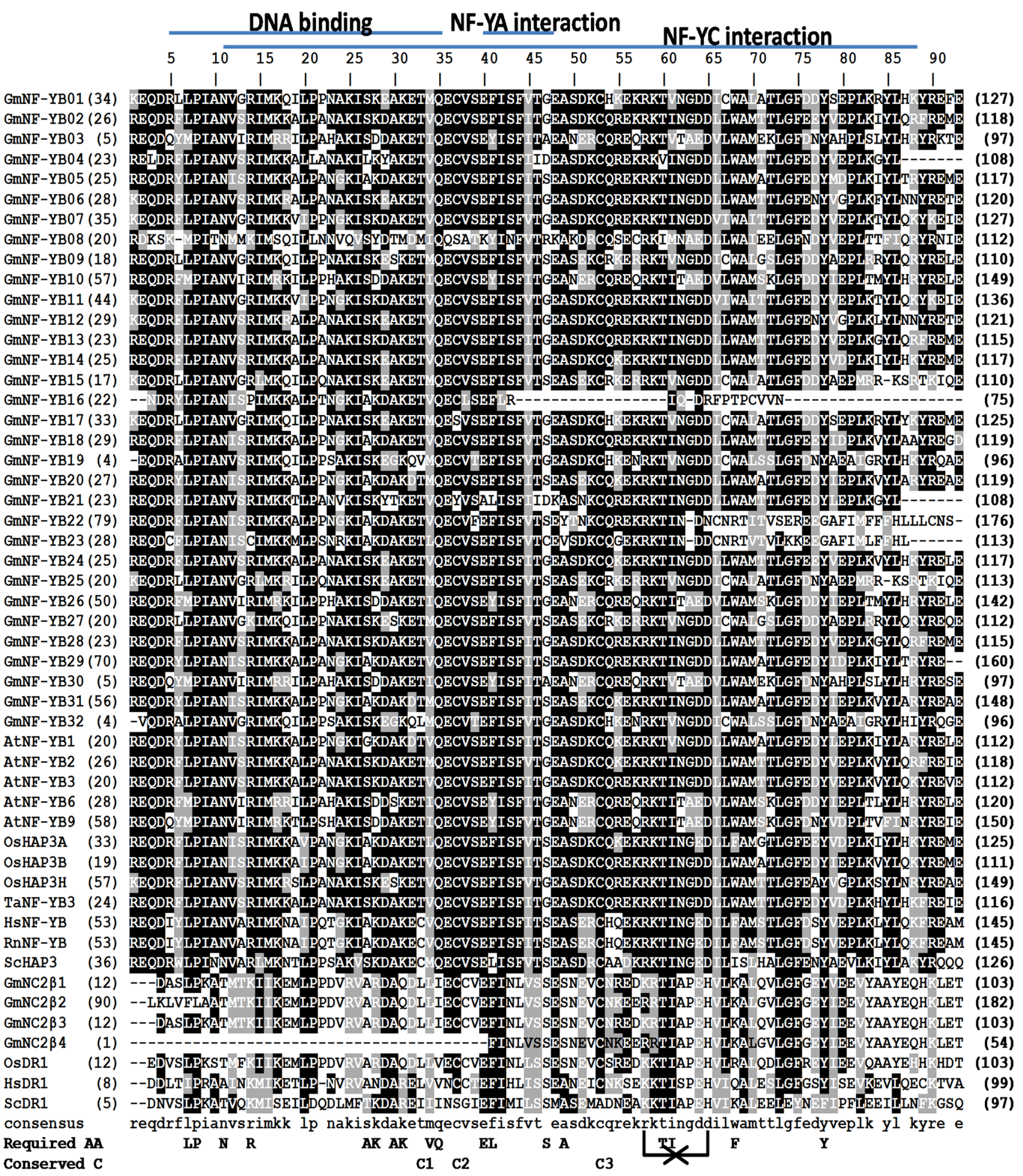

Fig. 5 Alignment of soybean NF-YB domains. The referred sequences are from Arabidopsis (At), rice (Os), wheat (Ta), human $(\mathrm{Hs})$, mouse $(\mathrm{Rn})$ and yeast $(\mathrm{Sc})$. Numbers in parentheses are the actual amino acid numbers of the start and end of NF-YB domain. Required amino acids (Required AA) are important for DNA bind- ing and subunit interaction from S. cerevisiae (Xing et al. 1993) and rat (Maity and de Crombrugghe 1992). Conserved cysteine C1, C2, $\mathrm{C} 3$ of NF-YB and the putative H-bonds between arginine $(\mathrm{R})$ and aspartate (D) were indicated in the bottom line. $\mathrm{NC} 2 \beta$ (or DR1) group which is homologous to NF-YB are included 


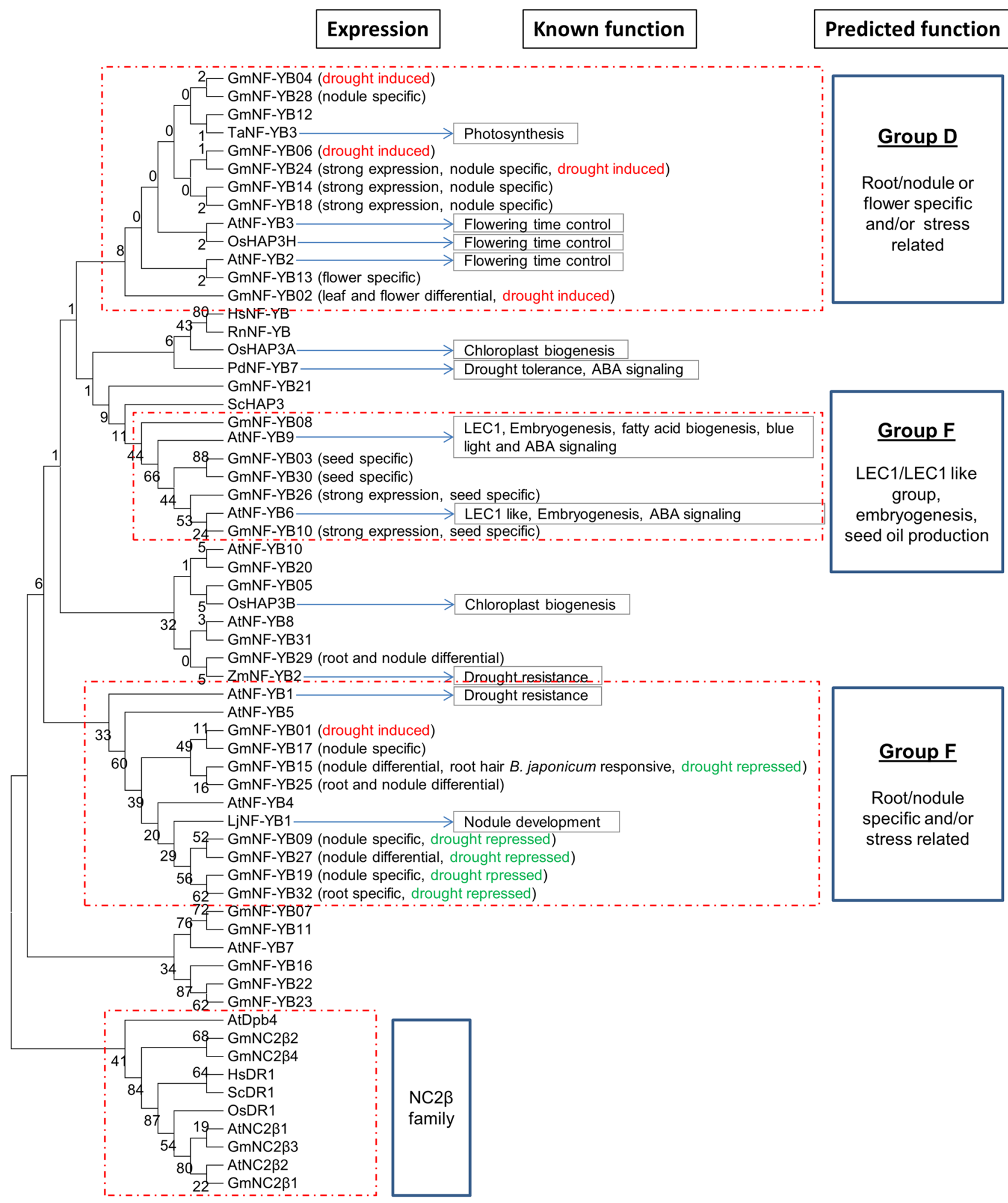

Fig. 6 Phylogenetic tree of soybean NF-YB. Function prediction for soybean genes were based on gene expression and homology to the referred characterized proteins which are written in bold for AtNFYB2 and AtNF-YB3 (Kumimoto et al. 2008), AtNF-YB6 (Lotan et al.
1998; Kwong et al. 2003; Lee et al. 2003; Tan et al. 2011), AtNFYB9 (Kwong et al. 2003; Lee et al. 2003; Warpeha et al. 2007), OSHAP3 (Miyoshi et al. 2003), AtNF-YB1 and ZmNF-YB2 (Nelson et al. 2007) and LjNF-YB1 (Soyano et al. 2013) 
accurate for lineage inferences. Although a crucial unit of NF-Y complex, less is known about the function of NF-YC proteins in plants. Limited work in Arabidopsis and Medicago showed that individual NF-YC is involved in nodule development, flowering control, ER stress response and ABA response in seed germination. Although the sequences are highly similar, the functions of previously characterized genes within tree branches appear too diverse (Fig. 8) to be used to assign a function within the NF-YC family. Group $\mathrm{G}$ includes genes with diverse functions relating to flower time control, stress adaptation and development (Ben-Naim et al. 2006; Wenkel et al. 2006). In Arabidopsis, NF-YC3, NF-YC4 and NF-YC9 are required for the CONSTANSmediated, photoperiod-dependent regulation of the flowering locus FT (Kumimoto et al. 2010). The soybean members in group $\mathrm{G}$ also share homology with the Arabidopsis AtNFYC2, which controls ER stress adaptation via unfolded protein response (Liu and Howell 2010), and the common bean (Phaseolus vulgaris) PvNF-YC1 which has been identified to positively regulate symbiotic interaction between the plant and the rhizobia (Zanetti et al. 2010). PvNF-YC1 activates cortical cell divisions and promotes nodule development in response to bacterial infection. Another close ortholog, Picea wilsonii PwHAP5, on the other hand, is involved in pollen tube development (Yu et al. 2011). In contrast, genes belonging to group $\mathrm{H}$ have protein sequences very conserved and possibly function in seed development due to specific expression in seeds and share strong homology to an Arabidopsis seed development-related gene AtNF-YC12. It is important to note that two soybean GmNF-YCO8 and GmNF-YC12 are induced by the inoculation with $B$. japonicum indicating that these two genes might be specific to soybean response to the inoculation.

\section{$N C 2$}

Our analysis also shows that the $\mathrm{NC} 2 \alpha$ and Dpb groups share some homology to NF-YC proteins and NC2 $\beta$ shares homology to NF-YB (Petroni et al. 2012). Although there is sequence similarity, NF-YC cannot pair with $\mathrm{NC} 2 \beta$ and NF-YB cannot pair with NC2 $\alpha$ (Zemzoumi et al. 1999). This binding preference might be explained by the difference in sequence structure, specifically the W44 of NF-YC at the end of helix alpha2 (Fig. 7), which is not present in $\mathrm{NC} 2 \alpha$. Additional to this difference, the hydrophobic cores organizing L1 and L2 regions are also different from that of NC2 (Romier et al. 2003). Although it is sharing some homology, all NC2 and Dpb3/Dpb4 genes are classified in a separate clade from phylogenetic tree analysis (Figs. 6, 8). In addition, $\mathrm{NC} 2$ and $\mathrm{Dpb} 3 / \mathrm{Dpb} 4$ appear to have different functions such as NC2 associates with TATA boxbinding protein to bind TATA box in the core promoters and the Dpb4/Dpb4 complex with DNA polymerase $\varepsilon$ and the chromatin-remodeling complex CHRAC (Kamada et al. 2001; Hartlepp et al. 2005).

\section{Discussion}

In the present report, we attempted to incorporate all the current annotations to explore the soybean NF-Y genes. Based on phylogenetics and up to date annotations, we identify 21 NF-YA, 32 NF-YB and 15 NF-YC and assign putative roles in plant growth and development, rhizobiumsoybean symbiosis and drought adaptation for each subgroups. The updated clarification between NC2, Dpb3/4 and NF-Y (Petroni et al. 2012) allows accurate classification of NF-Y genes and updates databases and literature of previously characterized ones in plants including $A$. thaliana, O. sativa, T. aestivum, Brachypodium distachyon, Selaginella moellendorffii and Brassica napus (Stephenson et al. 2007; Thirumurugan et al. 2008; Siefers et al. 2009; Cao et al. 2011; Liang et al. 2013; Saha et al. 2013). Although there is high primary sequence similarity among these gene families, $\mathrm{NC} 2$ and $\mathrm{Dpb} 3 / 4$ groups are the outliners in the reported analyses from wheat, Arabidopsis and $B$. distachyon (Stephenson et al. 2007; Siefers et al. 2009; Cao et al. 2011). There is also a distinct functionality of NC2 groups: NC2 complex works as a global transcriptional repressor that targets TBP, thereby blocking formation of the transcription preinitiation complex (Kim et al. 1997). To this end, it is also necessary to update latest annotations for proper classification for the soybean transcription databases that are currently active (Guo et al. 2008; Mochida et al. 2010; Wang et al. 2010). With the recent reports describing the involvement of NF-Y in nodule and seed development, and nitrogen-fixing bacterium interactions (Combier et al. 2006; Libault et al. 2010; Severin et al. 2010; Soyano et al. 2013; Battaglia et al. 2014; Laloum et al. 2014; Ripodas et al. 2014; Singh et al. 2014) and our current transcriptional analysis under drought treatments, our analyses suggest possible roles of soybean NF-Y genes in seed and nodule development and drought responses.

Plant fertility, embryogenesis and seed development

The involvement of NF-Y genes in developmental processes, particularly seed development and embryogenesis, has been identified in all three subfamilies (Petroni et al. 2012; Laloum et al. 2013). As expected, our transcript analysis identified several groups of soybean NF-YA, NF-YB and NF-YC which have highly specific or differential expression in soybean seeds and close relationship to the known genes. GmNF-FA subfamily appears to have more genes ( 8 out of total 16 GmNF-YA genes with available expression data) highly and differentially expressed 


\section{DNA binding}

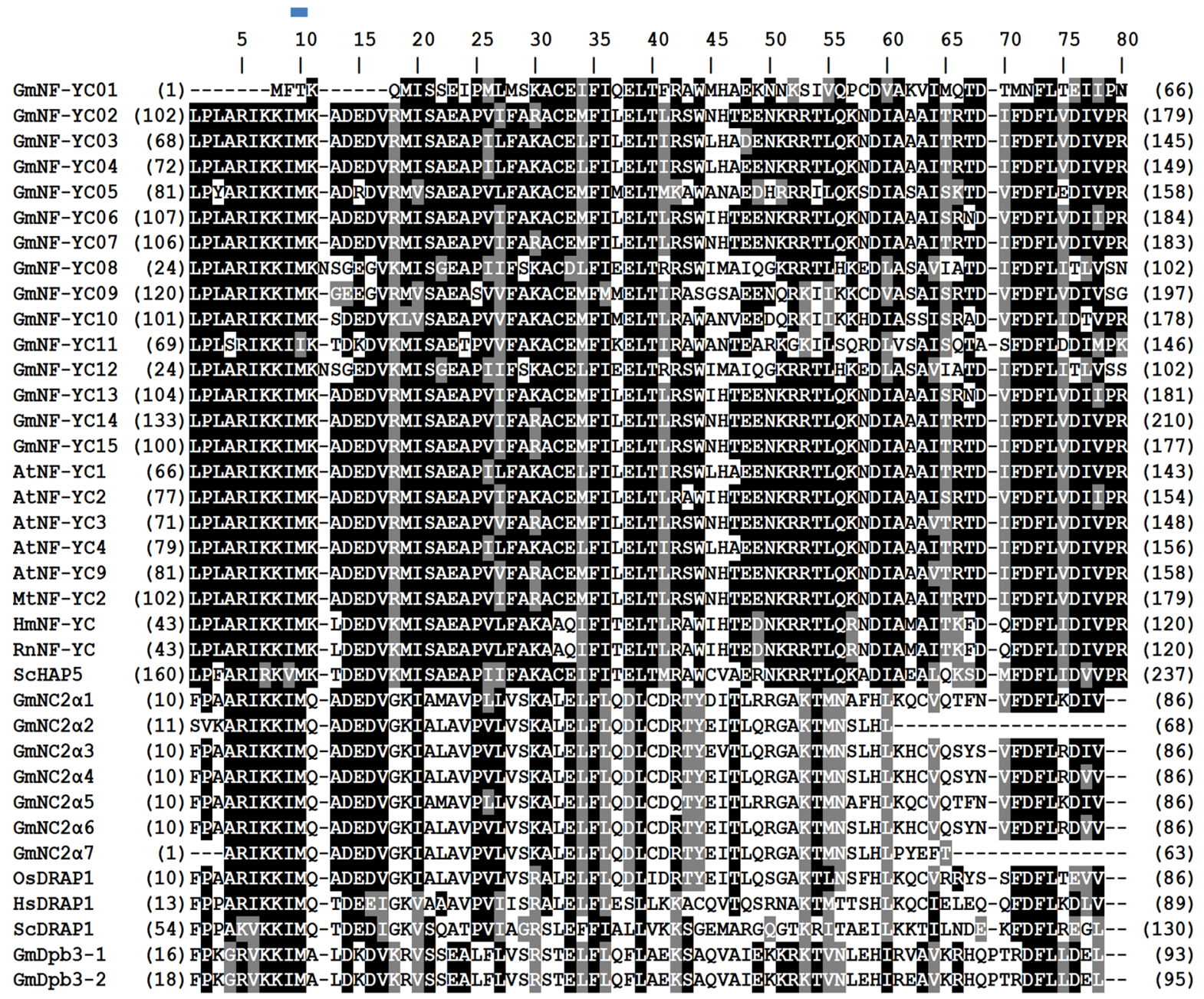

consensus lplarikkimk adedvrmisaeapvlfakaceifi eLtlrsw teenkrrtlqk dia aitrtd ifdflvdivpr Required AA AR KKI $\quad$ L A $\quad$ I $\quad$ L $\quad I \quad A \quad L r$

Fig. 7 Alignment of soybean NF-YC domains. The referred sequences are from Arabidopsis (At), Medicago (Mt), human (Hs), mouse $(\mathrm{Rn})$ and yeast $(\mathrm{Sc})$. Numbers in parentheses are the actual amino acid numbers of the start and end of domain NF-YC. Required amino acids (Required AA) are important for DNA binding and subu- nit interaction from yeast (Xing et al. 1993) and rat (Maity and de Crombrugghe 1992). The putative H-bonds between arginine (R) and aspartate (D) were indicated in the bottom line. NC2 $\alpha$ and Dpb (or DRAP1) groups which are homologous to NF-YC are included

GmNF-YB30, Figs. 5, 6) are highly similar to the Arabidopsis AtLEC1 and L1L (Meinke 1992; West et al. 1994; Parcy et al. 1997; Lotan et al. 1998; Kwong et al. 2003; Junker et al. 2012). LEC1 and L1L are particularly different from other NF-YB proteins and appear to possess unique residues that are important to regulate hormonal and light signaling during embryogenesis (Lee et al. 2003; Junker et al. 2012). Indeed, their orthologs in other plant species showed the highly conserved function in regulation of the transition from embryo to adult status (Zhang et al. 2002; Yazawa et al. 2004; Alemanno et al. 2008; Mu et al. 2008; 


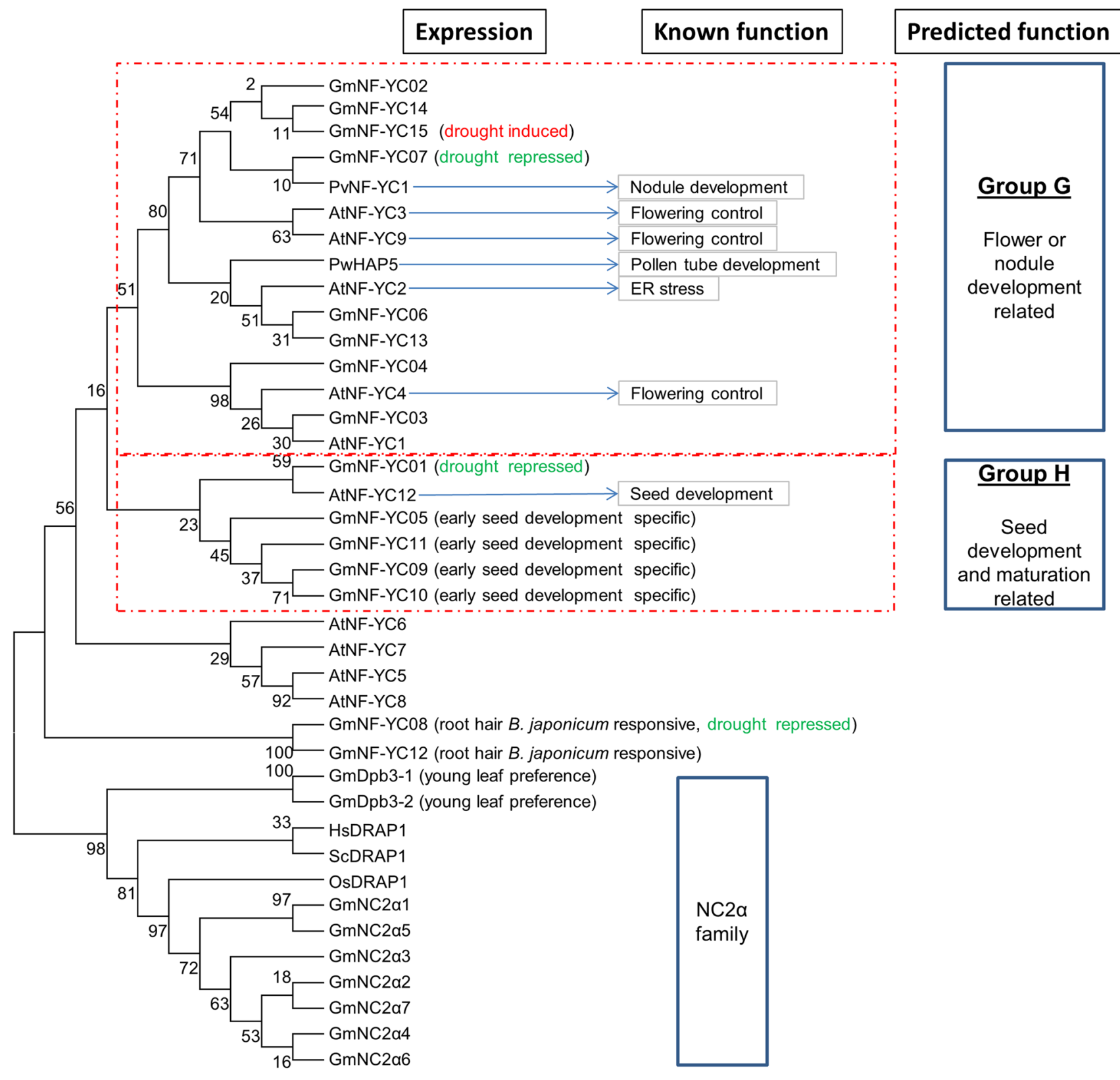

Fig. 8 Phylogenetic tree of soybean NF-YC. Function prediction for soybean genes was based on gene expression and homology to the referred characterized proteins which are written in bold for AtNF-

Schellenbaum et al. 2008; Shen et al. 2010; Cao et al. 2011; Tan et al. 2011). In addition, overexpression of LEC1, L1L and their orthologs resulted in seed oils via activation of various genes regulating fatty acid biosynthesis $(\mathrm{Mu}$ et al. 2008; Shen et al. 2010; Tan et al. 2011). Seed-specific expression is also seen in soybean GmNF-YC group for NF-YC05, NF-YC09, NF-YC10, NF-YC11. These genes share sequence similarity with the Arabidopsis AtNFYC12, a flower differentially expressed gene (Siefers et al. 2009). During seed developmental stages, AtNF-YC12 is
YC2 (Liu and Howell 2010), AtNF-YC3, AtNF-YC4 and AtNFYC9 (Kumimoto et al. 2010), PvNF-YC1 (Zanetti et al. 2010) and PwHAP5 (Yu et al. 2011)

highly expressed in chalazal endosperm and chalazal seed coat of green mature seed (http://www.seedgenenetwork. net/). AtNF-YC12 interacts bilaterally with LEC1 and L1L in yeast-two hybrid assays (Hackenberg et al. 2012) indicating that they co-regulate seed development and embryogenesis. Therefore, it would be interesting to investigate the functions of soybean gene combinations in the control of embryogenesis and seed oil production in soybean.

Increasing evidences have showed that NF-Y genes are key regulators of flowering time control in plants. 
Arabidopsis AtNF-YB2 and AtNF-YB3 promote flowering in response to inductive long-day condition (Kumimoto et al. 2008) through regulation of expression of FLOWERING LOCUS T (FT), a key gene that controls vegetative to floral meristem conversion (Mathieu et al. 2007). AtNFYB2 and AtNF-YB3 interact with three AtNF-YC (NFYC3, NF-YC4 and NF-YC9), which physically interact with CONSTANS (CO), and are required for $\mathrm{CO}$-mediated floral promotion to induce FT expression in the phloem tissue of long-day young leaves (Kumimoto et al. 2010). CO protein shares some homology to the Arabidopsis NF-YA subunits. Since overexpression of NF-YA1 and NF-YA4 caused delayed flowering, which may due to the impaired formation of NF-YB/NF-YC/CO (Wenkel et al. 2006). It is noted that the genes required for flowering time control often expressed in leaf vascular where $\mathrm{CO}$ genes are expressed (Kumimoto et al. 2010; Cao et al. 2011). Among the soybean NF-Y genes that are differentially expressed in young leaf, GmNF-YBO2, GmNF-YB13, GmNF-YCO4, GmNF-YCO6 and GmNF-YC14 are particularly of interest for their role in regulation of flowering time in soybean due to close sequence relationship with Arabidopsis and rice flowering time control genes AtNF-YB2, AtNF-YB3, OsHAP3H, AtNF-YC3 and AtNF-YC9 (Miyoshi et al. 2003; Kumimoto et al. 2008, 2010).

\section{Nitrogen nutrition and nodulation}

Nodulation is the result of a mutualistic interaction between the plant and symbiotic soil bacteria rhizobia, initiated by the infection of plant root hair cells by the symbiont. Initially, flavonoids secreted by the plant lead to the synthesis and secretion of a bacterial Nod factor (NF), which composes of chitooligosaccharide. Upon perception of NF, the symbiotic system activates various factors, of which, the nodule inception (NIN) and its downstream gene early nodulin (ERN) transcription factors are required for initiating nodulation-specific symbiotic processes (Madsen et al. 2010). In L. japonicus, NIN activates of the NF-Y subunit genes, $L j N F-Y A l$ and $L j N F-Y B I$ to induce cortical cell division, which is an initial step in root nodule organogenesis (Soyano et al. 2013). Similarly, in M. truncatula, the MtNFYA1 (MtHAP2-1) gene which shares homology to LjNFYA1 is important in nodule development and plant-bacterial symbiotic interaction (Combier et al. 2006; Laporte et al. 2013; Laloum et al. 2014). It is highly expressed in mature nodules, first induced at the onset of symbiotic development during preparation for, and initiation and progression of, symbiotic infection. MtNF-YA1 controls rhizobial infection progression by regulating the formation and the wall of infection thread. In M. truncatula, MtNF-YA1 and MtNFYA2 have a redundant function in nodulation development via ERN1 activation (Laloum et al. 2014). Our analysis in soybean shows that the $M$. truncatula MtNF-YA1 and $L$. japonicus LjNF-YA1 share strong homology to several soybean NF-YA genes (group A: GmNF-YAO1, GmNF$Y A 03, G m N F-Y A 10$ and GmNF-YA21). These genes are expressed specifically in nodules and induced by $B$. japonicum (Fig. 4), indicating a function in the symbiotic interaction and nodule development. We also found that genes in group F of GmNF-YB subfamily are specifically expressed in nodule and share a high sequence homology to L. japonicus LjNF-YB1 (Fig. 6), which regulates nodule development in interaction with LjNF-YA1, downstream of NIN (Soyano et al. 2013). Within this group, GmNY-YB15 is of particular interest for its nodule differential and rhizobium induced, suggesting a role in initial root nodule organogenesis. Members of subfamily NF-YC, on the other hand, are least known for the involvement in the nodulation development process. Among 15 soybean NF-YC genes, only GmNF-YC08 is induced by B. japonicum treatments (Supplementary Figure S2), suggesting an involvement in initial process of nodule development. Up to date, the Phaseolus vulgaris PvNF-YC1 is the first and the only NF-YC known to be required, and plays a key role in the nodule organogenesis and rhizobium infection (Zanetti et al. 2010; Mazziotta et al. 2013). Overexpression of $P v N F-Y C l$ improves nodulation efficiency on the less-efficient rhizobium strains, possibly through activation of the genes in G2/M transition. NF-YC1 could interact physically with SIN1, a GRAS-type transcription factor family which is required for efficient nodule development.

Stress response and adaptation

Increasing evidences have shown that individual NF-Y subunits, particularly NF-YA and NF-YB, are important to regulate drought and ER stress tolerance in both dicots and monocots with various mechanisms including tolerance to reduced water loss and low tissue water potential (Nelson et al. 2007; Li et al. 2008; Liu and Howell 2010; Han et al. 2013; Ni et al. 2013). The present investigation identified a large number of soybean NF-YA and NF-YB genes responsive to drought treatments and possibly involved in drought adaptation (Figs. 1, 2). Soybean NF-YA family appears to have more genes induced by drought ( 7 of 21 genes). Most these genes belong to the groups that have differential expression in the seeds, suggesting that they are involved in maturation and dehydration signaling. Three soybean genes: GmNF-YA09, GmNF-YA17 and GmNFYA19 have sequence homology to the drought tolerance GmNF-YA12 [or GmNF-YA3 (Ni et al. 2013)], AtNF-YA5 and AtNF-YA6 proteins ( $\mathrm{Li}$ et al. 2008; Fornari et al. 2013; $\mathrm{Mu}$ et al. 2013). GmNF-YA12 was induced by abscisic acid (ABA) and abiotic stresses, such as $\mathrm{NaCl}$ and cold, and has a role in drought tolerance by reduced leaf water loss 
via regulation of the ABA signaling pathway. AtNF-YA5 is induced by $\mathrm{ABA}, \mathrm{NaCl}$, cold and PEG treatment, and control water loss in Arabidopsis (Li et al. 2008). AtNF-YA5 also has a redundant role with AtNF-YA1, AtNF-YA6 and AtNF-YA9 which are downstream of LEC1 and regulate of embryogenesis and seed development in Arabidopsis (Mu et al. 2013). Most of drought-inducible soybean genes (5/7 genes) in this group are predicted to be targets of microRNA169 (data not shown), which is known to cleave GmNF-YA12 and AtNF-YA5 in response to drought stress (Li et al. 2008; Ni et al. 2013), indicating a conserved function in seed development and drought-response regulation.

Our phylogenetic analysis shows that two clades of soybean homology to the drought-tolerant Arabidopsis AtNF-YB1 and maize ZmNF-YB2 (Nelson et al. 2007). AtNF-YB1 and ZmNF-YB2 function in drought tolerance by maintaining leaf water potential, stomatal conductance and photosynthesis (Nelson et al. 2007). Surprisingly, most of the genes sharing homology to AtNF-YB1 (group F) are repressed by drought treatment. The majority of these genes are in fact specific/differential to nodule. Therefore, it is unlikely that they are involved in drought tolerance in soybean. GmNF-YB01, the only homolog of droughttolerant genes AtNF-YB1 and ZmNF-YB2, is expressed ubiquitously in various tissues and induced by drought (Fig. 6) suggesting that it is potentially involved in drought tolerance.

We found that the majority of genes that are highly specific to nodules are downregulated by drought stress. Soybean nodulation and nodule activity are strongly reduced under the stress (Sinclair et al. 2007) suggesting that NF-Y transcription factors are important in the regulation of nodule activity. Further characterization of these transcription factors will be useful in understanding of molecular regulation of nodulation and improvement of nitrogen assimilation during drought stress, as maintenance of nitrogen fixation is highly associated with maintenance of soybean productivity under the stress (Sinclair et al. 2007).

Acknowledgments Funding supports from Missouri Soybean Merchandising Council (MSMC) to the soybean genomics drought research program at the University of Missouri are gratefully appreciated.

Open Access This article is distributed under the terms of the Creative Commons Attribution License which permits any use, distribution, and reproduction in any medium, provided the original author(s) and the source are credited.

\section{References}

Alam MM, Tanaka T, Nakamura H, Ichikawa H, Kobayashi K, Yaeno T, Yamaoka N, Shimomoto K, Takayama K, Nishina H, Nishiguchi M (2014) Overexpression of a rice heme activator protein gene (OsHAP2E) confers resistance to pathogens, salinity and drought, and increases photosynthesis and tiller number. Plant Biotechnol J. doi:10.1111/pbi.12239

Alemanno L, Devic M, Niemenak N, Sanier C, Guilleminot J, Rio M, Verdeil JL, Montoro P (2008) Characterization of leafy cotyledon1-like during embryogenesis in Theobroma cacao L. Planta 227:853-866

Arents G, Moudrianakis EN (1995) The histone fold: a ubiquitous architectural motif utilized in DNA compaction and protein dimerization. Proc Natl Acad Sci USA 92:11170-11174

Aryee MJ, Gutierrez-Pabello JA, Kramnik I, Maiti T, Quackenbush J (2009) An improved empirical bayes approach to estimating differential gene expression in microarray time-course data: BETR (Bayesian Estimation of Temporal Regulation). BMC Bioinformatics 10:409

Battaglia M, Ripodas C, Clua J, Baudin M, Aguilar OM, Niebel A, Zanetti ME, Blanco FA (2014) A nuclear factor Y interacting protein of the GRAS family is required for nodule organogenesis, infection thread progression, and lateral root growth. Plant Physiol 164:1430-1442

Ben-Naim O, Eshed R, Parnis A, Teper-Bamnolker P, Shalit A, Coupland G, Samach A, Lifschitz E (2006) The CCAAT binding factor can mediate interactions between CONSTANS-like proteins and DNA. Plant J 46:462-476

Calvenzani V, Testoni B, Gusmaroli G, Lorenzo M, Gnesutta N, Petroni K, Mantovani R, Tonelli C (2012) Interactions and CCAAT-binding of Arabidopsis thaliana NF-Y subunits. PLoS One 7:e42902

Cao S, Kumimoto RW, Siriwardana CL, Risinger JR, Holt BF 3rd (2011) Identification and characterization of NF-Y transcription factor families in the monocot model plant Brachypodium distachyon. PLoS One 6:e21805

Ceribelli M, Dolfini D, Merico D, Gatta R, Viganò AM, Pavesi G, Mantovani R (2008) The histone-like NF-Y is a bifunctional transcription factor. Mol Cell Biol 28:2047-2058

Combier JP, Frugier F, de Billy F, Boualem A, El-Yahyaoui F, Moreau S, Vernie T, Ott T, Gamas P, Crespi M, Niebel A (2006) MtHAP2-1 is a key transcriptional regulator of symbiotic nodule development regulated by microRNA169 in Medicago truncatula. Genes Dev 20:3084-3088

Fornari M, Calvenzani V, Masiero S, Tonelli C, Petroni K (2013) The Arabidopsis NF-YA3 and NF-YA8 genes are functionally redundant and are required in early embryogenesis. PLoS One 8:e82043

Frontini M, Imbriano C, Manni I, Mantovani R (2004) Cell cycle regulation of NF-YC nuclear localization. Cell Cycle 3:217-222

Guo AY, Chen X, Gao G, Zhang H, Zhu QH, Liu XC, Zhong YF, Gu X, He K, Luo J (2008) PlantTFDB: a comprehensive plant transcription factor database. Nucleic Acids Res 36:D966-D969

Hackenberg D, Wu Y, Voigt A, Adams R, Schramm P, Grimm B (2012) Studies on differential nuclear translocation mechanism and assembly of the three subunits of the Arabidopsis thaliana transcription factor NF-Y. Mol Plant 5:876-888

Han X, Tang S, An Y, Zheng DC, Xia XL, Yin WL (2013) Overexpression of the poplar NF-YB7 transcription factor confers drought tolerance and improves water-use efficiency in Arabidopsis. J Exp Bot 64:4589-4601

Hartlepp KF, Fernandez-Tornero C, Eberharter A, Grune T, Muller CW, Becker PB (2005) The histone fold subunits of Drosophila CHRAC facilitate nucleosome sliding through dynamic DNA interactions. Mol Cell Biol 25:9886-9896

Hawkins RL, Nakamura M (1999) Expression of human growth hormone by the eukaryotic alga, Chlorella. Curr Microbiol 38:335-341

Hu R, Fan C, Li H, Zhang Q, Fu YF (2009) Evaluation of putative reference genes for gene expression normalization in soybean by quantitative real-time RT-PCR. BMC Mol Biol 10:93 
Jian B, Liu B, Bi Y, Hou W, Wu C, Han T (2008) Validation of internal control for gene expression study in soybean by quantitative real-time PCR. BMC Mol Biol 9:59

Junker A, Monke G, Rutten T, Keilwagen J, Seifert M, Thi TM, Renou JP, Balzergue S, Viehover P, Hahnel U, Ludwig-Muller J, Altschmied L, Conrad U, Weisshaar B, Baumlein H (2012) Elongation-related functions of LEAFY COTYLEDON1 during the development of Arabidopsis thaliana. Plant J 71:427-442

Kahle J, Baake M, Doenecke D, Albig W (2005) Subunits of the heterotrimeric transcription factor NF-Y are imported into the nucleus by distinct pathways involving importin beta and importin 13. Mol Cell Biol 25:5339-5354

Kamada K, Shu F, Chen H, Malik S, Stelzer G, Roeder RG, Meisterernst M, Burley SK (2001) Crystal structure of negative cofactor 2 recognizing the TBP-DNA transcription complex. Cell 106:71-81

Kim I-S, Sinha S, De Crombrugghe B, Maity SN (1996) Determination of functional domains in the $\mathrm{C}$ subunit of the CCAAT-binding factor $(\mathrm{CBF})$ necessary for formation of a CBF-DNA complex: CBF-B interacts simultaneously with both the CBF-A and $\mathrm{CBF}-\mathrm{C}$ subunits to form a heterotrimeric $\mathrm{CBF}$ molecule. Mol Cell Biol 16:4003-4013

Kim S, Na JG, Hampsey M, Reinberg D (1997) The Dr1/DRAP1 heterodimer is a global repressor of transcription in vivo. Proc Natl Acad Sci USA 94:820-825

Kumimoto RW, Adam L, Hymus GJ, Repetti PP, Reuber TL, Marion CM, Hempel FD, Ratcliffe OJ (2008) The nuclear factor Y subunits NF-YB2 and NF-YB3 play additive roles in the promotion of flowering by inductive long-day photoperiods in Arabidopsis. Planta 228:709-723

Kumimoto RW, Zhang Y, Siefers N, Holt BF 3rd (2010) NF-YC3, NF-YC4 and NF-YC9 are required for CONSTANS-mediated, photoperiod-dependent flowering in Arabidopsis thaliana. Plant J 63:379-391

Kwong RW, Bui AQ, Lee H, Kwong LW, Fischer RL, Goldberg RB, Harada JJ (2003) LEAFY COTYLEDON1-LIKE defines a class of regulators essential for embryo development. Plant Cell 15:5-18

Laloum T, De Mita S, Gamas P, Baudin M, Niebel A (2013) CCAATbox binding transcription factors in plants: Y so many? Trends Plant Sci 18:157-166

Laloum T, Baudin M, Frances L, Lepage A, Billault-Penneteau B, Cerri MR, Ariel F, Jardinaud MF, Gamas P, de Carvalho-Niebel F, Niebel A (2014) Two CCAAT box-binding transcription factors redundantly regulate early steps of the legume-rhizobia endosymbiosis. Plant J 79:757-768

Laporte P, Lepage A, Fournier J, Catrice O, Moreau S, Jardinaud MF, Mun JH, Larrainzar E, Cook DR, Gamas P, Niebel A (2013) The CCAAT box-binding transcription factor NF-YA1 controls rhizobial infection. J Exp Bot 65(2):481-494

Le DT, Aldrich DL, Valliyodan B, Watanabe Y, Ha CV, Nishiyama R, Guttikonda SK, Quach TN, Gutierrez-Gonzalez JJ, Tran LS, Nguyen HT (2012) Evaluation of candidate reference genes for normalization of quantitative RT-PCR in soybean tissues under various abiotic stress conditions. PLoS One 7:e46487

Lee H, Fischer RL, Goldberg RB, Harada JJ (2003) Arabidopsis LEAFY COTYLEDON1 represents a functionally specialized subunit of the CCAAT binding transcription factor. Proc Natl Acad Sci USA 100:2152-2156

Levesque-Lemay M, Albani D, Aldcorn D, Hammerlindl J, Keller W, Robert LS (2003) Expression of CCAAT-binding factor antisense transcripts in reproductive tissues affects plant fertility. Plant Cell Rep 21:804-808

Li WX, Oono Y, Zhu J, He XJ, Wu JM, Iida K, Lu XY, Cui X, Jin H, Zhu JK (2008) The Arabidopsis NFYA5 transcription factor is regulated transcriptionally and posttranscriptionally to promote drought resistance. Plant Cell 20:2238-2251

Li L, Yu Y, Wei J, Huang G, Zhang D, Liu Y, Zhang L (2013) Homologous HAP5 subunit from Picea wilsonii improved tolerance to salt and decreased sensitivity to ABA in transformed Arabidopsis. Planta 238:345-356

Liang M, Yin X, Lin Z, Zheng Q, Liu G, Zhao G (2013) Identification and characterization of NF-Y transcription factor families in Canola (Brassica napus L.). Planta 239:107-126

Libault M, Thibivilliers S, Bilgin D, Radwan O, Benitez M, Clough S, Stacey G (2008) Identification of four soybean reference genes for gene expression normalization. Plant Genome 1:44-54

Libault M, Farmer A, Brechenmacher L, Drnevich J, Langley RJ, Bilgin DD, Radwan O, Neece DJ, Clough SJ, May GD, Stacey G (2010) Complete transcriptome of the soybean root hair cell, a single-cell model, and its alteration in response to Bradyrhizobium japonicum infection. Plant Physiol 152:541-552

Liu JX, Howell SH (2010) bZIP28 and NF-Y transcription factors are activated by ER stress and assemble into a transcriptional complex to regulate stress response genes in Arabidopsis. Plant Cell 22:782-796

Lotan T, Ohto M, Yee KM, West MAL, Lo R, Kwong RW, Yamagishi K, Fischer RL, Goldberg RB, Harada JJ (1998) Arabidopsis LEAFY COTYLEDON1 is sufficient to induce embryo development in vegetative cells. Cell 93:1195-1205

Madsen LH, Tirichine L, Jurkiewicz A, Sullivan JT, Heckmann AB, Bek AS, Ronson CW, James EK, Stougaard J (2010) The molecular network governing nodule organogenesis and infection in the model legume Lotus japonicus. Nat Commun 1:10

Maity SN, de Crombrugghe B (1992) Biochemical analysis of the B subunit of the heteromeric CCAAT-binding factor. A DNA-binding domain and a subunit interaction domain are specified by two separate segments. J Biol Chem 267:8286-8292

Mantovani R (1998) A survey of 178 NF-Y binding CCAAT boxes. Nucleic Acids Res 26:1135-1143

Mantovani R (1999) The molecular biology of the CCAAT-binding factor NF-Y. Gene 239:15-27

Mathieu J, Warthmann N, Kuttner F, Schmid M (2007) Export of FT protein from phloem companion cells is sufficient for floral induction in Arabidopsis. Curr Biol 17:1055-1060

Mazziotta L, Reynoso MA, Aguilar OM, Blanco FA, Zanetti ME (2013) Transcriptional and functional variation of NF-YC1 in genetically diverse accessions of Phaseolus vulgaris during the symbiotic association with Rhizobium etli. Plant Biol (Stuttg) $15: 808-818$

Meinke DW (1992) A homoeotic mutant of Arabidopsis thaliana with leafy cotyledons. Science 258:1647-1650

Mendes A, Kelly AA, van Erp H, Shaw E, Powers SJ, Kurup S, Eastmond PJ (2013) bZIP67 regulates the omega-3 fatty acid content of Arabidopsis seed oil by activating fatty acid desaturase3. Plant Cell 25:3104-3116

Miyoshi K, Ito Y, Serizawa A, Kurata N (2003) OsHAP3 genes regulate chloroplast biogenesis in rice. Plant J 36:532-540

Mochida K, Yoshida T, Sakurai T, Yamaguchi-Shinozaki K, Shinozaki K, Tran L-SP (2010) LegumeTFDB: an integrative database of Glycine max, Lotus japonicus and Medicago truncatula transcription factors. Bioinformatics 26:290-291

Mu J, Tan H, Zheng Q, Fu F, Liang Y, Zhang J, Yang X, Wang T, Chong K, Wang XJ, Zuo J (2008) LEAFY COTYLEDON1 is a key regulator of fatty acid biosynthesis in Arabidopsis. Plant Physiol 148:1042-1054

Mu J, Tan H, Hong S, Liang Y, Zuo J (2013) Arabidopsis transcription factor genes NF-YA1, 5, 6, and 9 play redundant roles in male gametogenesis, embryogenesis, and seed development. Mol Plant 6:188-201 
Nelson DE, Repetti PP, Adams TR, Creelman RA, Wu J, Warner DC, Anstrom DC, Bensen RJ, Castiglioni PP, Donnarummo MG, Hinchey BS, Kumimoto RW, Maszle DR, Canales RD, Krolikowski KA, Dotson SB, Gutterson N, Ratcliffe OJ, Heard JE (2007) Plant nuclear factor Y (NF-Y) B subunits confer drought tolerance and lead to improved corn yields on water-limited acres. Proc Natl Acad Sci USA 104:16450-16455

Ni Z, Hu Z, Jiang Q, Zhang H (2013) GmNFYA3, a target gene of miR169, is a positive regulator of plant tolerance to drought stress. Plant Mol Biol 82:113-129

Parcy F, Valon C, Kohara A, Misera S, Giraudat J (1997) The ABSCISIC ACID-INSENSITIVE3, FUSCA3, and LEAFY COTYLEDON1 loci act in concert to control multiple aspects of Arabidopsis seed development. Plant Cell 9:1265-1277

Peng WT, Lee YW, Nester EW (1998) The phenolic recognition profiles of the Agrobacterium tumefaciens VirA protein are broadened by a high level of the sugar binding protein ChvE. J Bacteriol 180:5632-5638

Petroni K, Kumimoto RW, Gnesutta N, Calvenzani V, Fornari M, Tonelli C, Holt BF 3rd, Mantovani R (2012) The promiscuous life of plant NUCLEAR FACTOR Y transcription factors. Plant Cell 24:4777-4792

Ramakers C, Ruijter JM, Deprez RHL, Moorman AF (2003) Assumption-free analysis of quantitative real-time polymerase chain reaction (PCR) data. Neurosci Lett 339:62-66

Ripodas C, Clua J, Battaglia M, Baudin M, Niebel A, Zanetti ME, Blanco F (2014) Transcriptional regulators of legume-rhizobia symbiosis: nuclear factors Ys and GRAS are two for tango. Plant Signal Behav 9:e28847

Romier C, Cocchiarella F, Mantovani R, Moras D (2003) The NF-YB/NF-YC structure gives insight into DNA binding and transcription regulation by CCAAT factor NF-Y. J Biol Chem 278:1336-1345

Saha J, Gupta K, Gupta B (2013) In silico characterization and evolutionary analyses of CCAAT binding proteins in the lycophyte plant Selaginella moellendorffi genome: A growing comparative genomics resource. Comput Biol Chem 47:81-88

Schellenbaum P, Jacques A, Maillot P, Bertsch C, Mazet F, Farine S, Walter B (2008) Characterization of VvSERK1, VvSERK2, VvSERK3 and VvL1L genes and their expression during somatic embryogenesis of grapevine (Vitis vinifera L.). Plant Cell Rep 27:1799-1809

Schmutz J, Cannon SB, Schlueter J, Ma J, Mitros T, Nelson W, Hyten DL, Song Q, Thelen JJ, Cheng J, Xu D, Hellsten U, May GD, Yu Y, Sakurai T, Umezawa T, Bhattacharyya MK, Sandhu D, Valliyodan B, Lindquist E, Peto M, Grant D, Shu S, Goodstein D, Barry K, Futrell-Griggs M, Abernathy B, Du J, Tian Z, Zhu L, Gill N, Joshi T, Libault M, Sethuraman A, Zhang XC, Shinozaki K, Nguyen HT, Wing RA, Cregan P, Specht J, Grimwood J, Rokhsar D, Stacey G, Shoemaker RC, Jackson SA (2010) Genome sequence of the palaeopolyploid soybean. Nature 463:178-183

Severin AJ, Woody JL, Bolon YT, Joseph B, Diers BW, Farmer AD, Muehlbauer GJ, Nelson RT, Grant D, Specht JE, Graham MA, Cannon SB, May GD, Vance CP, Shoemaker RC (2010) RNA-seq atlas of Glycine max: a guide to the soybean transcriptome. BMC Plant Biol 10:160

Shen B, Allen WB, Zheng P, Li C, Glassman K, Ranch J, Nubel D, Tarczynski MC (2010) Expression of ZmLEC1 and ZmWRI1 increases seed oil production in maize. Plant Physiol 153:980-987

Siefers N, Dang KK, Kumimoto RW, Bynum WE 4th, Tayrose G, Holt BF (2009) Tissue-specific expression patterns of Arabidopsis NF-Y transcription factors suggest potential for extensive combinatorial complexity. Plant Physiol 149:625-641
Sinclair TR, Purcell LC, King CA, Sneller CH, Chen P, Vadez V (2007) Drought tolerance and yield increase of soybean resulting from improved symbiotic $\mathrm{N}_{2}$ fixation. Field Crops Res 101:68-71

Singh S, Katzer K, Lambert J, Cerri M, Parniske M (2014) CYCLOPS, a DNA-binding transcriptional activator, orchestrates symbiotic root nodule development. Cell Host Microbe 15:139-152

Sinha S, Kim IS, Sohn KY, De Crombrugghe B, Maity SN (1996) Three classes of mutations in the A subunit of the CCAATbinding factor $\mathrm{CBF}$ delineate functional domains involved in the three-step assembly of the CBF-DNA complex. Mol Cell Biol 16:328-337

Soyano T, Kouchi H, Hirota A, Hayashi M (2013) Nodule inception directly targets NF-Y subunit genes to regulate essential processes of root nodule development in Lotus japonicus. PLoS Genet 9:e1003352

Srivastava GP, Xu D (2007) Genome-scale probe and primer design with PRIMEGENS. Methods Mol Biol 402:159-176

Stephenson TJ, McIntyre CL, Collet C, Xue GP (2007) Genome-wide identification and expression analysis of the NF-Y family of transcription factors in Triticum aestivum. Plant Mol Biol 65:77-92

Stephenson TJ, McIntyre CL, Collet C, Xue GP (2011) TaNF-YB3 is involved in the regulation of photosynthesis genes in Triticum aestivum. Funct Integr Genomics 11:327-340

Tamura K, Peterson D, Peterson N, Stecher G, Nei M, Kumar S (2011) MEGA5: molecular evolutionary genetics analysis using maximum likelihood, evolutionary distance, and maximum parsimony methods. Mol Biol Evol 28:2731-2739

Tan H, Yang X, Zhang F, Zheng X, Qu C, Mu J, Fu F, Li J, Guan R, Zhang H, Wang G, Zuo J (2011) Enhanced seed oil production in canola by conditional expression of Brassica napus LEAFY COTYLEDON1 and LEC1-LIKE in developing seeds. Plant Physiol 156:1577-1588

Thirumurugan T, Ito Y, Kubo T, Serizawa A, Kurata N (2008) Identification, characterization and interaction of HAP family genes in rice. Mol Genet Genomics 279:279-289

Thon M, Al Abdallah Q, Hortschansky P, Scharf DH, Eisendle M, Haas H, Brakhage AA (2010) The CCAAT-binding complex coordinates the oxidative stress response in eukaryotes. Nucleic Acids Res 38:1098-1113

Vandesompele J, De Preter K, Pattyn F, Poppe B, Van Roy N, De Paepe A, Speleman F (2002) Accurate normalization of real-time quantitative RT-PCR data by geometric averaging of multiple internal control genes. Genome Biol 3:RESEARCH0034

Wang Z, Libault M, Joshi T, Valliyodan B, Nguyen H, Xu D, Stacey G, Cheng J (2010) SoyDB: a knowledge database of soybean transcription factors. BMC Plant Biol 10:14

Warpeha KM, Upadhyay S, Yeh J, Adamiak J, Hawkins SI, Lapik YR, Anderson MB, Kaufman LS (2007) The GCR1, GPA1, PRN1, NF-Y signal chain mediates both blue light and abscisic acid responses in Arabidopsis. Plant Physiol 143:1590-1600

Wei X, Xu J, Guo H, Jiang L, Chen S, Yu C, Zhou Z, Hu P, Zhai H, Wan J (2010) DTH8 suppresses flowering in rice, influencing plant height and yield potential simultaneously. Plant Physiol 153:1747-1758

Wenkel S, Turck F, Singer K, Gissot L, Le Gourrierec J, Samach A, Coupland G (2006) CONSTANS and the CCAAT box binding complex share a functionally important domain and interact to regulate flowering of Arabidopsis. Plant Cell 18:2971-2984

West MA, Yee KM, Danao J, Zimmerman JL, Fischer RL, Goldberg RB, Harada JJ (1994) LEAFY COTYLEDON1 is an essential regulator of late embryogenesis and cotyledon identity in Arabidopsis. Plant Cell Online 6:1731-1745

Xing Y, Fikes JD, Guarente L (1993) Mutations in yeast HAP2/HAP3 define a hybrid CCAAT box binding domain. EMBO J 12:4647 
Yazawa K, Takahata K, Kamada H (2004) Isolation of the gene encoding Carrot leafy cotyledon 1 and expression analysis during somatic and zygotic embryogenesis. Plant Physiol Biochem 42:215-223

Yu Y, Li Y, Huang G, Meng Z, Zhang D, Wei J, Yan K, Zheng C, Zhang L (2011) PwHAP5, a CCAAT-binding transcription factor, interacts with PwFKBP12 and plays a role in pollen tube growth orientation in Picea wilsonii. J Exp Bot 62:4805-4817

Zanetti ME, Blanco FA, Beker MP, Battaglia M, Aguilar OM (2010) A C subunit of the plant nuclear factor NF-Y required for rhizobial infection and nodule development affects partner selection in the common bean-Rhizobium etli symbiosis. Plant Cell 22:4142-4157
Zemzoumi K, Frontini M, Bellorini M, Mantovani R (1999) NF-Y histone fold alpha1 helices help impart CCAAT specificity. J Mol Biol 286:327-337

Zhang S, Wong L, Meng L, Lemaux PG (2002) Similarity of expression patterns of knotted1 and ZmLEC1 during somatic and zygotic embryogenesis in maize (Zea mays L.). Planta 215:191-194

Zhao M, Ding H, Zhu JK, Zhang F, Li WX (2011) Involvement of miR169 in the nitrogen-starvation responses in Arabidopsis. New Phytol 190:906-915 